\title{
De Finetti Lattices and Magog Triangles
}

\author{
Andrew Beveridge \\ Department of Mathematics, Statistics and Computer Science \\ Macalester College \\ St Paul, Minnesota, U.S.A. \\ abeverid@macalester.edu \\ Ian Calaway \\ Department of Economics \\ Stanford University \\ Stanford, California, U.S.A. \\ icalaway@stanford.edu \\ Kristin Heysse \\ Department of Mathematics, Statistics and Computer Science \\ Macalester College \\ St Paul, Minnesota, U.S.A. \\ kheysse@macalester.edu
}

Submitted: Dec 27, 2019; Accepted: Dec 26, 2020; Published: Feb 12, 2021

(C) The authors. Released under the CC BY-ND license (International 4.0).

\begin{abstract}
The order ideal $B_{n, 2}$ of the Boolean lattice $B_{n}$ consists of all subsets of size at most 2. Let $F_{n, 2}$ denote the poset refinement of $B_{n, 2}$ induced by the rules: $i<j$ implies $\{i\} \prec\{j\}$ and $\{i, k\} \prec\{j, k\}$. We give an elementary bijection from the set $\mathcal{F}_{n, 2}$ of linear extensions of $F_{n, 2}$ to the set of shifted standard Young tableau of shape $(n, n-1, \ldots, 1)$, which are counted by the strict-sense ballot numbers. We find a more surprising result when considering the set $\mathcal{F}_{n, 2}^{1}$ of minimal poset refinements in which each singleton is comparable with all of the doubletons. We show that $\mathcal{F}_{n, 2}^{1}$ is in bijection with magog triangles, and therefore is equinumerous with alternating sign matrices. We adopt our proof techniques to show that row reversal of an alternating sign matrix corresponds to a natural involution on gog triangles.
\end{abstract}

Mathematics Subject Classifications: 05A10, 05A15 


\section{Introduction}

\subsection{Overview}

The Boolean lattice $B_{n}$ consists of subsets of $[n]=\{1,2, \ldots, n\}$ ordered by inclusion. The order ideal $B_{n, 2} \subset B_{n}$ consists of all subsets of size at most 2 . We investigate two families of poset refinements of the Boolean lattice $B_{n, 2}$. The first family is in bijection with shifted standard Young tableau of staircase shape $(n, n-1, \ldots, 1)$. The second family is in bijection with magog triangles. We assume familiarity with standard notions from poset theory, as found in Chapter 3 of Stanley [30]; a helpful collection of poset terminology is found in Section 1.3.1 below.

Define $F_{n, 2}$ to be the poset refinement of $B_{n, 2}$ where we add the relations

(R1) $\{i\} \prec\{k\}$ if and only if $i<k$.

(R2) If $i<j$ and $k<\ell$ then

$$
\{i, j\} \prec\{k, \ell\} \quad \text { if and only if } \quad(i<k \text { and } j \leqslant \ell) \text { or }(i \leqslant k \text { and } j<\ell) \text {. }
$$

Transitivity of the set inclusion relation $\{i\} \prec\{i, j\}$ and (R2) yields the relation

$$
\{i\} \prec\{k, \ell\} \quad \text { if and only if } i<\min \{k, \ell\} \text {. }
$$

The Hasse diagrams for $B_{n, 2}$ and $F_{n, 2}$ are shown in Figure 1.
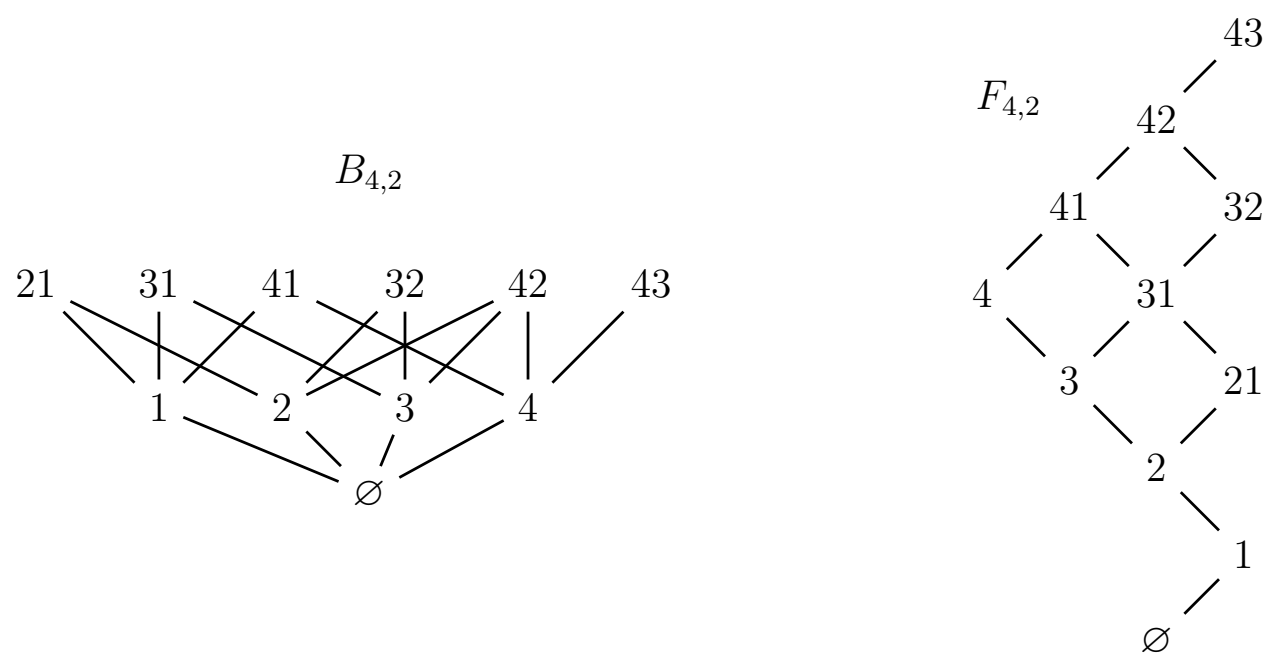

Figure 1: Hasse diagrams for the order ideal $B_{n, 2}$ and the de Finetti lattice $F_{n, 2}$. The sets $\{i\}$ and $\{j, k\}$ are denoted by $i$ and $j k$ where $j>k$.

It is straightforward to confirm that the poset $F_{n, 2}$ is a distributive lattice. First, we show every pair of subsets $x, y \in F_{n, 2}$ has a meet (greatest lower bound) $x \wedge y$ and a join 
(least upper bound) $x \vee y$. For $j>i$ and $\ell>k$, we have

$$
\begin{aligned}
\{j\} \wedge\{\ell\} & =\{\min \{j, \ell\}\}, & \{j\} \vee\{\ell\} & = & \{\max \{j, \ell\}\}, \\
\{j\} \wedge\{\ell, k\} & =\{\min \{j, \ell\}\}, & \{j\} \vee\{\ell, k\} & = & \{\max \{j, \ell\}, k\}, \\
\{j, i\} \wedge\{\ell, k\} & =\{\min \{j, \ell\}, \min \{i, k\}\}, & \{j, i\} \vee\{\ell, k\} & = & \{\max \{j, \ell\}, \max \{i, k\}\} .
\end{aligned}
$$

Note that if we identify $\{j\}$ with $\{j, 0\}$ and $\{\ell\}$ with $\{\ell, 0\}$, then the meet and join rules for doubleton pairs imply the other rules. Moreover, it then becomes easy to confirm that $F_{n, 2}$ is a distributive lattice: $x \vee(y \wedge z)=(x \vee y) \wedge(x \vee z)$ and $x \wedge(y \vee z)=(x \wedge y) \vee(x \wedge z)$.

We refer to $F_{n, 2}$ as the de Finetti lattice; the origin of this name will be illuminated in Section 1.2 below. We resolve two questions concerning families of poset refinements of $F_{n, 2}$.

Definition 1. The set $\mathcal{F}_{n, 2}$ is the collection of linear extensions of $F_{n, 2}$.

We give a simple bijection between the total orders in $\mathcal{F}_{n, 2}$ and shifted standard Young tableau (shifted SYT) of shape $(n, n-1, \ldots, 1)$, see OEIS A003121 [29]. In these shifted SYT of staircase shape, the first box in row $i>1$ is located below the second box of row $i-1$. The integers $1,2, \ldots, n(n+1) / 2$ are arranged in the boxes so that the rows and the columns are both increasing. These are equinumerous with the number of strict-sense ballots with $n$ candidates, where candidate $k$ receives $k$ votes, and during the vote count, candidate $k$ always leads candidate $\ell$ for $n \geqslant k>\ell \geqslant 1$, see [3]. The strict-sense ballot number sequence begins with

$$
1,1,2,12,286,33592,23178480, \ldots
$$

and the general formula for the $n$th strict-sense ballot number is

$$
\left(\begin{array}{c}
n+1 \\
2
\end{array}\right) ! \frac{\prod_{k=1}^{n-1} k !}{\prod_{k=1}^{n}(2 k-1) !}
$$

The proof of the following proposition appears in Section 2.

Proposition 2. The set $\mathcal{F}_{n, 2}$ is in bijection with shifted standard Young tableaux of shape $(n, n-1, \ldots, 1)$. Therefore $\mathcal{F}_{n, 2}$ is enumerated by the strict-sense ballot numbers.

Our second family of poset refinements is less conventional.

Definition 3. The set $\mathcal{F}_{n, 2}^{1}$ is the collection of poset refinements of $F_{n, 2}$ that are induced by resolving all disjoint pairs of the form $\{i\},\{k, \ell\}$.

The seven posets in $\mathcal{F}_{4,2}^{1}$ are shown in Figure 2. Two of these posets are linear extensions. In the remaining five refinements, the doubletons 41 and 32 are incomparable. There are two ways to resolve this relation: either $41 \prec 32$ or $32 \prec 41$. This accounts for the 12 linear extensions in $\mathcal{F}_{n, 2}$, in accordance with Proposition 2. We prove the following lemma in Section 1.2. 


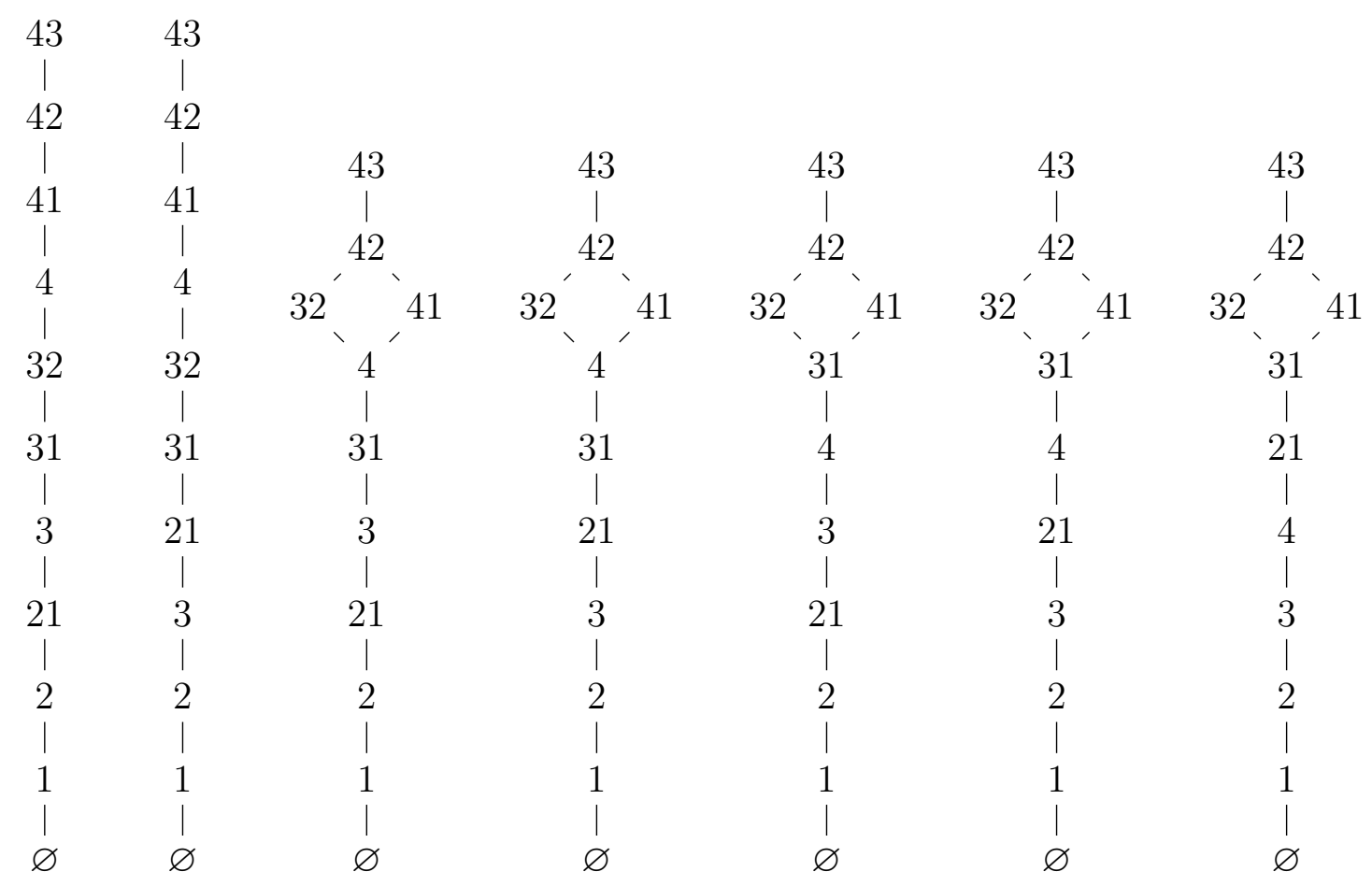

Figure 2: Hasse diagrams for the seven posets in $\mathcal{F}_{4,2}^{1}$.

Lemma 4. Every poset in $\mathcal{F}_{n, 2}^{1}$ is a lattice.

Our main result is a bijection between $\mathcal{F}_{n, 2}^{1}$ and magog triangles of size $n-1$, see OEIS A005130 [29]. Magog triangles are a family of triangular integer arrays which are in bijection with totally symmetric self-complimentary plane partitions (TSSCPPs). Their counterpart gog triangles are in bijection with alternating sign matrices (ASMs). Gog and magog triangles were instrumental to Zeilberger's famous proof that ASMs are equinumerous with TSSCPPs [36]. The first numbers in this sequence are

$$
1,2,7,42,429,7436,218348, \ldots
$$

and the general formula is

$$
\prod_{k=0}^{n-1} \frac{(3 k+1) !}{(n+k) !} .
$$

We use $\mathcal{M}_{n}$ and $\mathcal{G}_{n}$ to denote the respective families of magog triangles and gog triangles of size $n$. Here is our main theorem, which we prove in Section 3.

Theorem 5. The family $\mathcal{F}_{n, 2}^{1}$ of de Finetti refinements of the lattice $F_{n, 2}$ is in bijection with the family $\mathcal{M}_{n-1}$ of magog triangles of size $n-1$.

We make two remarks about our proof of Theorem 5. First, our bijection makes use of a new triangular family that is in bijection with magog triangles, though we defer the 
description of these kagog triangles to Section 3.1. Second, we view these triangular arrays of positive integers as pyramids of cubes colored gray and white, adhering to appropriate stacking rules. This geometric viewpoint is essential to our proof, which employs an affine transformation and a color inversion to turn a kagog pyramid into a magog pyramid.

This two-color cube pyramid model may be useful to others interested in studying the enigmatic relationship between ASMs and TSSCPPs. As an example of its potential utility, we prove the following theorem in Section 4.

Theorem 6. Reversing the order of the rows of $n \times n$ ASMs induces an involution on gog triangles $\mathcal{G}_{n}$. The corresponding involution on two-color cube pyramids reverses the cube coloring and performs a rigid transformation of the pyramid.

\subsection{De Finetti Lattices}

In this section, we motivate the study of $F_{n, 2}$ and its poset refinements. We also prove Lemma 4. In various settings, (including probability, computational algebra, and social choice theory) researchers have investigated total orders of the power set $\mathcal{P}([n])$ satisfying the following two conditions:

(F1) $\varnothing \prec\{1\} \prec\{2\} \prec \ldots \prec\{n\}$, and

(F2) $X \prec Y$ if and only if $X \cup Z \prec Y \cup Z$ for all $Z \subset[n]$ such that $(X \cup Y) \cap Z=\varnothing$.

Condition (F1) is the canonical ordering of the singleton sets. Condition (F2) is de Finetti's axiom [12], which was first formulated in a probabilistic setting. This axiom can be restated as: $A \prec B$ if and only if $A \backslash B \prec B \backslash A$. Intuitively, condition (F2) states that when we have comparable sets, adding the same element (or elements) to both sets will not change the comparison. For $n \geqslant 3$, conditions (F1) and (F2) do not completely determine a total order; for example, we cannot deduce whether $\{1,2\} \prec\{3\}$ or $\{3\} \prec\{1,2\}$ from these first principles.

We make two observations about total orders satisfying these conditions. First, induction on $|B \backslash A|$ shows that if $A \subsetneq B$ then $A \prec B$. Second, another simple induction proof confirms that if $x_{i} \leqslant y_{i}$ for $1 \leqslant i \leqslant k$ then (F1) and (F2) lead to the (intuitive) conclusion that $\left\{x_{1}, x_{2}, \ldots, x_{k}\right\} \preceq\left\{y_{1}, y_{2}, \ldots, y_{k}\right\}$, where equality holds only when these sets are identical. In summary, we have a linear extension of the Boolean lattice that also extends the standard ordering on the integers $[n]$ to an ordering of the subsets of $[n]$.

Total orders of $\mathcal{P}([n])$ satisfying (F1) and (F2) appear under various names, including comparative probability orders, Boolean term orders, and completely separable preference orders, see OEIS A005806 [29]. Each of these names reflects the application setting rather than the defining properties of the total order. Therefore, we opt for the generic name de Finetti total order, paying homage to de Finetti's axiom. Furthermore, there is no harm in starting with the Boolean lattice $B_{n}$ rather than the set $\mathcal{P}([n])$, since the set inclusion relations are enforced by (F1) and (F2). 
Definition 7. A de Finetti refinement $\left(E, \prec_{E}\right)$ of the Boolean lattice $\left(B_{n}, \prec\right)$ is a poset refinement that adheres to (F1) and to (F2) for all sets $X, Y \subset[n]$ that are comparable in E. A de Finetti total order is a linear extension of $B_{n}$ that adheres to (F1) and (F2). The collection of de Finetti total orders of $B_{n}$ is denoted $\mathcal{F}_{n}$.

Note that (F2) does not require that all pairs $X$ and $Y$ are comparable, but when they are, the sets $X \cup Z$ and $Y \cup Z$ are also comparable, as are $X \backslash Y$ and $Y \backslash X$. The number $\left|\mathcal{F}_{n}\right|$ of de Finetti total orders for $1 \leqslant n \leqslant 7$ is

$$
1,1,2,14,546,169444,560043206
$$

but there is still no known general formula. Enumerations of the 14 de Finetti total orders for $n=4$ can be found in $[17,6,11]$. This current work germinated while studying de Finetti total orders: we restricted our attention to the order ideal $B_{n, 2} \subset B_{n}$ of subsets of size at most 2, and then considered the poset refinements of $B_{n, 2}$ that adhere to (F1) and (F2).

Definition 8. A de Finetti refinement $\left(E_{2}, \prec_{E_{2}}\right)$ of the order ideal $\left(B_{n, 2}, \prec\right)$ is a poset refinement that adheres to $(\mathrm{F} 1)$ and to $(\mathrm{F} 2)$ for all sets $X, Y$ that are comparable in $E_{2}$.

When restricting ourselves to $B_{n, 2}$, the conditions (F1) and (F2) simplify to the set inclusion relations (as noted above) plus the relations (R1) and (R2) from Section 1.1. Indeed, (F1) clearly implies (R1). As for (R2), let $i<j$ and $k<\ell$. If $i<k$ and $j \leqslant \ell$ then $\{i, j\} \prec\{k, j\} \preceq\{k, \ell\}$, so $\{i, j\} \prec\{k, \ell\}$ by transitivity. The case $i \leqslant k$ and $j<\ell$ proceeds similarly.

Having made this connection, we relate the results in Section 1.1 to de Finetti refinements. First, we now recognize the lattice $F_{n, 2}$ as the unique minimal de Finetti refinement of $B_{n, 2}$. Any de Finetti refinement of $B_{n, 2}$ must adhere to conditions (R1) and (R2), so it must contain the lattice $F_{n, 2}$. Second, any valid poset refinement of $F_{n, 2}$ is automatically a de Finetti refinement. The incomparable pairs $X, Y$ of $F_{n, 2}$ are disjoint and at least one must be a doubleton set, so $Z=\varnothing$ is the only allowed choice in (F2).

Let us revisit $\mathcal{F}_{n, 2}$ and $\mathcal{F}_{n, 2}^{1}$ in light of this observation. We can now see that the collection $\mathcal{F}_{n, 2}$ of linear extensions of $F_{n, 2}$ is also the collection of de Finetti total orders of $B_{n, 2}$. Likewise, the refinements of $\mathcal{F}_{n, 2}^{1}$ are de Finetti refinements of $B_{n, 2}$. As an aside, we find it useful to view $\mathcal{F}_{n, 2}^{1}$ as the collection of minimal de Finetti refinements of $B_{n, 2}$ among those in which the singleton sets are comparable with all other elements.

We now prove Lemma 4: each poset refinement in $\mathcal{F}_{n, 2}^{1}$ is a lattice.

Proof of Lemma 4. Consider any pair of elements $x, y \in F_{n, 2}$. Since $F_{n, 2}$ is a lattice, these elements have least upper bound $x \vee y$, and greatest lower bound $x \wedge y$ in $F_{n, 2}$.

Let $\left(P, \prec_{P}\right) \in \mathcal{F}_{n, 2}^{1}$. Since $\left(P, \prec_{P}\right)$ is a refinement of $\left(F_{n, 2}, \prec\right)$, we know that $x \vee y$ is still an upper bound of $x$ and $y$ in $P$ and that that $x \wedge y$ is still a lower bound of $x$ and $y$ in $P$.

We must show $x, y$ have a least upper bound $x \vee_{P} y$ and a greatest lower bound $x \wedge_{P} y$ in $P$. If at least one of $x, y$ is a singleton set, then $x$ and $y$ are comparable in $P$, which 
means that $x \vee_{P} y=\max \{x, y\}$ and $x \wedge_{P} y=\min \{x, y\}$. So we now assume that both $x$ and $y$ are doubleton sets.

Let us prove that $x$ and $y$ have a least upper bound in $P$. Assume for the sake of contradiction that $z \in P$ is a minimal $x, y$ upper bound that is incomparable with $x \vee y$ in $P$. Recall that the empty set and the singleton sets are comparable with every element in $P$, so both $z$ and $x \vee y$ must be doubleton sets. Furthermore, $z$ is an upper bound of at most one of $x$ and $y$ in $F_{n, 2}$ : otherwise $x \vee y \prec z$ in $F_{n, 2}$ and therefore $x \vee y \prec_{P} z$. There are two cases.

First, suppose that $z$ is $F_{n, 2}$-incomparable with both $x$ and $y$. Recall that the additional comparisons in $P$ are induced by resolving disjoint pairs $\{i\},\{j, k\}$. In order to be comparable in $P$, there must be singletons $s_{1}, s_{2}$ (not necessarily distinct) such that $x \prec_{P} s_{1} \prec_{P} z$ and $y \prec_{P} s_{2} \prec_{P} z$ in $P$. Without loss of generality, $s_{1} \leqslant s_{2}$, which means that $s_{2}$ is an $x, y$ upper bound in $P$ and $s_{2} \prec_{P} z$. This contradicts the minimality of $z$.

Second, suppose (without loss of generality) that $x \prec z$ in $F_{n, 2}$ while $y$ and $z$ are $F_{n, 2}$-incomparable. Analogous to the previous case, there must be a singleton $s$ such that $y \prec_{P} s \prec_{P} z$. Now, if $x \prec_{P} s$ then $s$ is an $x, y$ upper bound in $P$, contradicting the minimality of $z$. Otherwise, $s \prec_{P} x$ which means that $x=x \vee_{P} y$, which also contradicts the minimality of $z$.

The doubletons $x, y$ also have a greatest lower bound in $P$ : this proof is entirely parallel to the least upper bound proof.

This concludes our motivation for studying $\mathcal{F}_{n, 2}$ and $\mathcal{F}_{n, 2}^{1}$. Given our success at characterizing these refinements of $B_{n, 2}$, it is natural to formulate analogous research questions for de Finetti refinements of $B_{n, m}$. We defer those formulations to our concluding section.

\subsection{Related Work}

\subsubsection{The Boolean Lattice}

A partially ordered set (or poset for short) consists of a set $P$ and a binary relation $\preceq$ that is reflexive $(x \preceq x$ ), antisymmetric (if $x \preceq y$ and $y \preceq x$ then $x=y$ ) and transitive (if $x \preceq y$ and $y \preceq z$ then $x \preceq z$ ). An order ideal is a subset $I \subset P$ that is downward-closed: if $x \in I$ and $y \preceq x$ then $y \in I$. A lattice is a poset such that every pair of elements have a least upper bound and a greatest lower bound. We obtain a refinement of a poset $P$ by adding relations between pairs of incomparable elements of $P$. A total order is a poset in which every pair of elements is comparable. A linear extension of a poset $P$ is a refinement of $P$ that is a total order (equivalently: a linear extension is an order-preserving bijection $\sigma: P \rightarrow[N]$ where $|P|=N)$. See Chapter 3 of Stanley [30] for an introduction to posets and lattices.

For a poset $P$, let $\mathcal{L}(P)$ denote the set of linear extensions of $P$. Brightwell and Tetali [9] determined an accurate asymptotic formula for $\left|\mathcal{L}\left(B_{n}\right)\right|$, improving on work of Sha and Kleitman [27]. The value of $\left|\mathcal{L}\left(B_{n}\right)\right|$ is known for $1 \leqslant n \leqslant 7$, see OEIS A046873 [29]. The $n=7$ case was recently determined by Brower and Christensen [10] using machinery developed to study the game of Chomp played on the Boolean lattice. Pruesse and Ruskey 
[26] introduced the linear extension graph $G\left(B_{n}\right)$ whose vertex set is $\mathcal{L}\left(B_{n}\right)$ and whose edge set consists of pairs of linear extensions that differ by a single adjacent transposition. Felsner and Massow [13] determined the diameter of $G\left(B_{n}\right)$.

Researchers have also studied linear extensions of subposets of $B_{n}$, including the order ideal $B_{n, m}$ of subsets of size at most $m$. Fink and Gregor [15] determined the linear extension diameter of the subposet $B_{n}^{1, k}$ of $B_{n}$ that is induced by levels 1 and $k$. Brouwer and Christensen [10] determined that

$$
\left|\mathcal{L}\left(B_{n, 2}\right)\right|=\frac{n !\left(\left(\begin{array}{l}
n \\
2
\end{array}\right)+n\right) !}{\prod_{i=1}^{n}\left(i n-\left(\begin{array}{l}
i \\
2
\end{array}\right)\right)}=\left(\begin{array}{c}
n+1 \\
2
\end{array}\right) ! \frac{1}{\prod_{i=1}^{n}\left(n-\frac{i-1}{2}\right)}
$$

and computed $\left|\mathcal{L}\left(B_{n, 3}\right)\right|$ for $n \leqslant 7$. Comparing this formula with our Proposition 2 shows that $n ! \cdot\left|\mathcal{F}_{n, 2}\right|=o\left(\left|\mathcal{L}\left(B_{n, 2}\right)\right|\right)$. In other words, the de Finetti linear extensions of $B_{n, 2}$ are exceptionally rare.

\subsubsection{De Finetti Total Orders}

The de Finetti total orders $\mathcal{F}_{n}$ are total orders of $\mathcal{P}([n])$ that satisfy both (F1) and (F2). These total orders appear in a variety of settings with names that reflect the application at hand $[14,23,6,11]$. In probability theory, the total orders in $\mathcal{F}_{n}$ are known as comparative probability orders, and they enjoy applications in decision theory and economics [21, 14, 16, 28]. A comparative probability order $\preceq$ is additively representable when there is a probability measure $p:[n] \rightarrow[0,1]$ that induces the order, namely $p(X) \leqslant p(Y)$ if and only if $X \preceq Y$.

In a more algebraic context, Maclagan [23] refered to total orders in $\mathcal{F}_{n}$ as Boolean term orders and studied their combinatorial properties. Maclagan introduced a flip operation between Boolean term orders, which consists of multiple (related) adjacent transpositions so that (F2) still holds. The flip graph is the graph with vertex set $\mathcal{F}_{n}$, where two orders are adjacent when they differ by one flip. It is an open question whether the flip graph is connected for $n \geqslant 9$. Christian et al. [11] further studied flippable pairs of orders and their relation to the polytope of an additively representable order.

In social choice theory, these total orders are called completely separable preferences $[18,6]$. In this setting, de Finetti's condition ensures that a voter's preference for the outcomes on a subset $S \subset[n]$ of proposals is independent of the outcome of the proposals in $\bar{S}$. Hodge and TerHaar [20] showed that the number of de Finetti total orders satisfies $n ! \cdot\left|\mathcal{F}_{n}\right|=o\left(\mathcal{L}\left(B_{n}\right)\right)$. In fact, they proved the stronger condition that linear extensions with at least one pair $X, Y$ of proper nontrivial subsets satisfying condition (F2) are vanishingly rare. Other research on separable preferences focuses on the admissibility problem: which collection of subsets can occur as the collection of separable sets $S$, meaning that (F2) holds for any subsets $X, Y \subset S$ and any $Z \subset \bar{S}$, see [20, 19, 4].

\subsubsection{Gog Triangles and Magog Triangles}

Theorem 2 establishes a bijection between the de Finetti refinements $\mathcal{F}_{n, 2}^{1}$ and the magog triangles $\mathcal{M}_{n-1}$. This connects our poset refinement problem to the illustrious family of 
alternating sign matrices. See [7, 8], respectively, for a brief or an extended recounting of the history of the famous alternating sign matrix conjecture. The magog triangles $\mathcal{M}_{n}$ are in bijection with totally the symmetric self-complementary plane partitions (TSSCPP) in a $2 n \times 2 n \times 2 n$ box. Andrews [1] proved that the number of such TSSCPP is given by (1). Meanwhile, gog triangles $\mathcal{G}_{n}$ are in bijection with $n \times n$ alternating sign matrices (ASM). Zeilberger [36] proved that $\left|\mathcal{M}_{n}\right|=\left|\mathcal{G}_{n}\right|$, which confirmed that TSSCPPs and ASMs are equinumerous. Kuperberg [22] later gave a more streamlined proof using the 6 -vertex model from statistical mechanics.

There are many combinatorial manifestations of the ASM sequence $(1)$, see $[8,25]$. A natural bijective proof between TSSCPPs and ASMs (or equivalently, between magog and gog triangles) remains elusive, though progress on subfamilies has been achieved [2, 5, 33]. Posets and triangular arrays of numbers (such as gog, magog and kagog triangles) continue to play an essential role in ASM and TSSCPP research. Terwilliger [34] describes a poset refinement of the hypercube whose maximal chains are in bijection with alternating sign matrices (and gog triangles). Striker [32] defined a tetrahedral poset $T_{n}$ whose subposets trace connections between TSSCPPs, ASMs and other combinatorial sequences. In particular, $T_{n}$ has one subposet whose order ideals can be described via families of triangular arrays. The order ideals of one such subposet is in bijection with gog triangles (and hence with ASMs). There are six distinct subposets whose order ideals (with associated triangular families) are in bijection with magog triangles (and hence with TSSCPPs). We note that our kagog triangles are not among the triangular families described in [32], so the family of TSSCPP triangles continues to grow.

\section{Shifted Standard Young Tableau of Staircase Shape and $\mathcal{F}_{n, 2}$}

This brief section contains a proof of Proposition 2: we give a simple bijection between $\mathcal{F}_{n, 2}$ and shifted standard Young tableaux (shifted SYT) of shape $(n, n-1, \ldots, 1)$. Figure 3 exemplifies the mapping for $n=4$.

Proof of Proposition 2. To ease exposition, we identify the singleton $\{i\}$ with the doubleton $\{i, 0\}$. Ignore the set $\varnothing$ and lay out the lattice $F_{n, 2}$ in a shifted staircase grid so that row $k$ contains the sets $\{i, k-1\}$ for $k \leqslant i \leqslant n$ in increasing order. This grid induces a shifted staircase Ferrers diagram $(n, n-1, \ldots, 1)$ whose boxes are indexed the $n(n+1) / 2$ nontrivial members of $F_{n, 2}$.

Consider a total order $E \in \mathcal{F}_{n, 2}$. Place the integer $\ell$ in the box corresponding to the $\ell$ th set in total ordering $E$. The result is a shifted SYT of staircase shape: the rows and columns of the resulting tableau are both increasing because the total ordering satisfies properties (F1) and (F2) of Definition 7. This mapping is surjective: starting from a shifted SYT, we can reverse the process to find a total order $E \in \mathcal{F}_{n, 2}$ that maps to it. 


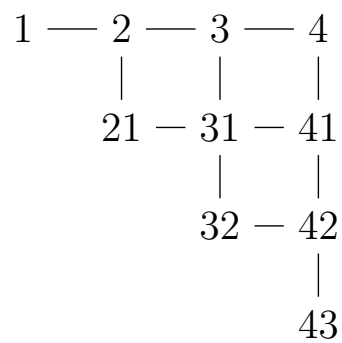

(a)

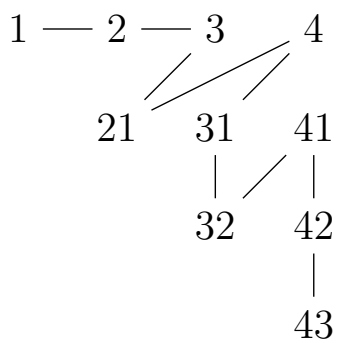

(b)

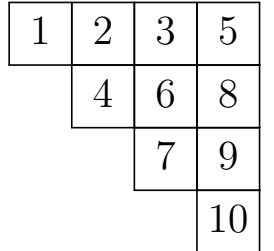

(c)

Figure 3: Mapping $F_{4,2}$ to a shifted standard Young tableau. (a) The nontrivial sets in $F_{4,2}$ laid out in a shifted staircase grid. (b) A de Finetti total order and (c) its corresponding shifted standard Young tableau.

\section{Kagog Triangles, Magog Triangles and $\mathcal{F}_{n, 2}^{1}$}

\subsection{Overview}

We outline the proof of Theorem 5, deferring the details to the subsections that follow. We begin by defining the new family of kagog triangles. This name is a variant of Zeilberger's gog and magog terminology, and is meant to invoke their connection to magog triangles.

Definition 9. A kagog triangle $K$ of index $n$ is an array of nonnegative integers $K(i, j)$ such that

(K1) $1 \leqslant j \leqslant i \leqslant n-1$, so the array is triangular;

(K2) $0 \leqslant K(i, j) \leqslant j$, so entries in column $j$ are at most $j$;

(K3) $K(i, j) \geqslant K(i+1, j)$, so columns are weakly decreasing; and

(K4) if $K(i, j)>0$ then $K(i, j+1)>K(i, j)$, so rows can start with multiple zeros, but then the positive values are strictly increasing.

We use $\mathcal{K}_{n}$ to denote the set of kagog triangles of index $n$.

The elements of $\mathcal{K}_{3}$ are

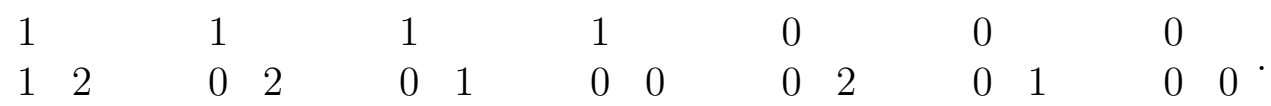

Note that a kagog triangle of index $n$ only has $n-1$ rows and columns. Our first theorem connects kagog triangles $\mathcal{K}_{n-1}$ to the poset refinements $\mathcal{F}_{n, 2}^{1}$.

Theorem 10. The set of de Finetti refinements $\mathcal{F}_{n, 2}^{1}$ is in bijection with the set of kagog triangles $\mathcal{K}_{n-1}$. 
Next, we turn our attention to the well-known family of magog triangles, which are in bijection with TSSCPPs.

Definition 11. A magog triangle $M$ of size $n$ is an array of positive integers $M(i, j)$ where

(M1) $1 \leqslant j \leqslant i \leqslant n$, so the array is triangular;

(M2) $1 \leqslant M(i, j) \leqslant j$, so entries in column $j$ are at most $j$;

(M3) $M(i, j) \leqslant M(i+1, j)$, so columns are weakly increasing; and

(M4) $M(i, j) \leqslant M(i, j+1)$, so rows are weakly increasing.

We use $\mathcal{M}_{n}$ to denote the set of magog triangles of size $n$.

When a magog triangle is viewed as a Gelfand-Tsetlin triangle of positive integers, conditions (M2) and (M4) are replaced by $M(j, j) \leqslant j$ and $M(i, j) \leqslant M(i+1, j+1)$, respectively. The elements of $\mathcal{M}_{3}$ are

$\begin{array}{llllllllllllllllllllll}1 & & & 1 & & & 1 & & & 1 & & & 1 & & & 1 & & & 1 & & \\ 1 & 1 & & 1 & 1 & & 1 & 1 & & 1 & 2 & & 1 & 1 & & 1 & 1 & & 1 & 2 & \\ 1 & 1 & 1 & 1 & 1 & 2 & 1 & 2 & 2 & 1 & 2 & 2 & 1 & 1 & 3 & 1 & 2 & 3 & 1 & 2 & 3\end{array}$.

Our second theorem addresses a duality between kagog triangles $\mathcal{K}_{n}$ and magog triangles $\mathcal{M}_{n}$.

Theorem 12. The set of magog triangles $\mathcal{M}_{n}$ is in bijection with the set of kagog triangles $\mathcal{K}_{n}$.

The magog triangles listed in (3) are ordered so that that they biject to the kagog triangles in (2). Also, note that we have chosen to left-justify our triangles (they are often presented using center alignment). This layout choice simplifies our geometric arguments. The key to proving Theorem 12 is to convert each of these triangles into a pyramid of stacked cubes, colored gray or white, so that gray cubes cannot appear above white cubes. We offer a generic definition for pyramid construction, which applies to any family $\mathcal{T}_{n}$ of triangular arrays that form a distributive lattice using the natural partial ordering $T_{1} \prec T_{2}$ whenever $T_{1}(i, j) \leqslant T_{2}(i, j)$ for $1 \leqslant j \leqslant i \leqslant n$. This includes magog triangles $\mathcal{M}_{n}$ and kagog triangles $\mathcal{K}_{n}$, as well as gog triangles $\mathcal{G}_{n}$ (defined in Section 4 below).

Definition 13. Let $\mathcal{T}_{n}$ be a finite distributive lattice of triangular arrays of positive integers $T=T(i, j)$ where $1 \leqslant j \leqslant i \leqslant n$ with minimal triangle $T_{\min }$ and maximal triangle $T_{\max }$. Define $\triangle T$ to be the two-color pyramid of cubes $(i, j, k)$ where $1 \leqslant i \leqslant j \leqslant n$ and $1 \leqslant k \leqslant T_{\max }(i, j)$ where the tower of cubes at $(i, j)$ consists of $T(i, j)$ white cubes below $T_{\max }(i, j)-T(i, j)$ gray cubes. Define $\triangle \mathcal{T}_{n}=\left\{\triangle T: T \in \mathcal{T}_{n}\right\}$ to be the collection of two-color pyramids. 

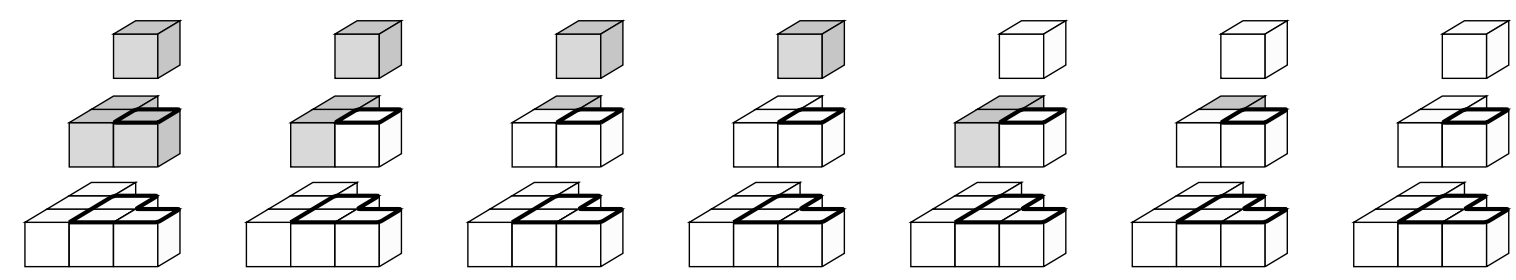

Figure 4: The two-color pyramids from $\triangle \mathcal{M}_{3}$, sliced into horizontal layers. The shadow of each layer is thickly drawn on the layer below. The pyramids are drawn using a righthanded coordinate system with the positive $x$-axis pointing out of the page.

Figure 4 shows the seven magog pyramids, listed in the same order as in (3). To facilitate visualization, the pyramids have been sliced into layers of equal height. This two-color pyramid mapping is a variation of the standard interpretation triangular array $T$ as a stack of cubes where the tower at $(i, j)$ has height $T(i, j)$. Indeed, we can view the white cubes as present and the gray cubes as absent. In our proof, tracking the absent cubes is essential, so the two-color pyramids are more illuminating. Intuitively, the bijection from magog triangles to kagog triangles corresponds to removing the bottom layer of the magog pyramid, then swapping the colors of the cubes and finally performing an appropriate affine transformation.

Proof of Theorem 5. Follows immediately from Theorem 10 and Theorem 12.

We prove Theorem 10 and Theorem 12 in the next two subsections.

\subsection{The bijection from $\mathcal{F}_{n, 2}^{1}$ to $\mathcal{K}_{n-1}$}

In this subsection, we prove Theorem 10. Figure 5 shows the de Finetti lattice $F_{n, 2}$ for $n=3,4,5$ and also indicates the sublattice

$$
I_{k}=\{\{j, i\}: 1 \leqslant i<j<k\}
$$

of doubletons that are incomparable with singleton $\{k\}$.

For $k \geqslant 3$, let $\Phi\left(I_{k}\right)$ be the collection of de Finetti refinements of $I_{k} \cup\{k\}$ for which the singleton $\{k\}$ is comparable with every doubleton of $I_{k}$ (and no additional extraneous relations). When we restrict a poset refinement $E \in \mathcal{F}_{n, 2}^{1}$ to the set $I_{k} \cup\{k\}$, we obtain some $E_{k} \in \Phi\left(I_{k}\right)$. Similarly, we can induce a unique poset refinement $E$ of $F_{n, 2}$ from a list $\left(E_{3}, E_{4}, \ldots, E_{n}\right)$ where $E_{k} \in \Phi\left(I_{k}\right)$. We will have $E \in \mathcal{F}_{n, 2}^{1}$ provided that the union of these orderings does not violate de Finetti's condition (F2). Figure 6 gives an example of a poset refinement $E \in \mathcal{F}_{n, 2}^{1}$ and its collection of $E_{k} \in \Phi\left(I_{k}\right)$.

Our bijection from the poset refinements of $F_{n, 2}$ in $\mathcal{F}_{n, 2}^{1}$ to the kagog triangles in $\mathcal{K}_{n-1}$ proceeds as follows. Given a de Finetti refinement $E \in \mathcal{F}_{n, 2}^{1}$, we create the corresponding list $\left(E_{3}, \ldots E_{n}\right)$ where $E_{k} \in \Phi\left(I_{k}\right)$. We then map refinement $E$ to a kagog triangle $K \in$ $\mathcal{K}_{n-1}$ so that refinement $E_{j}$ maps to row $j-2$ of triangle $K$ for $3 \leqslant j \leqslant n$. The row constraint (K3) of the kagog triangle will correspond to the internal structure of each $E_{k}$. 


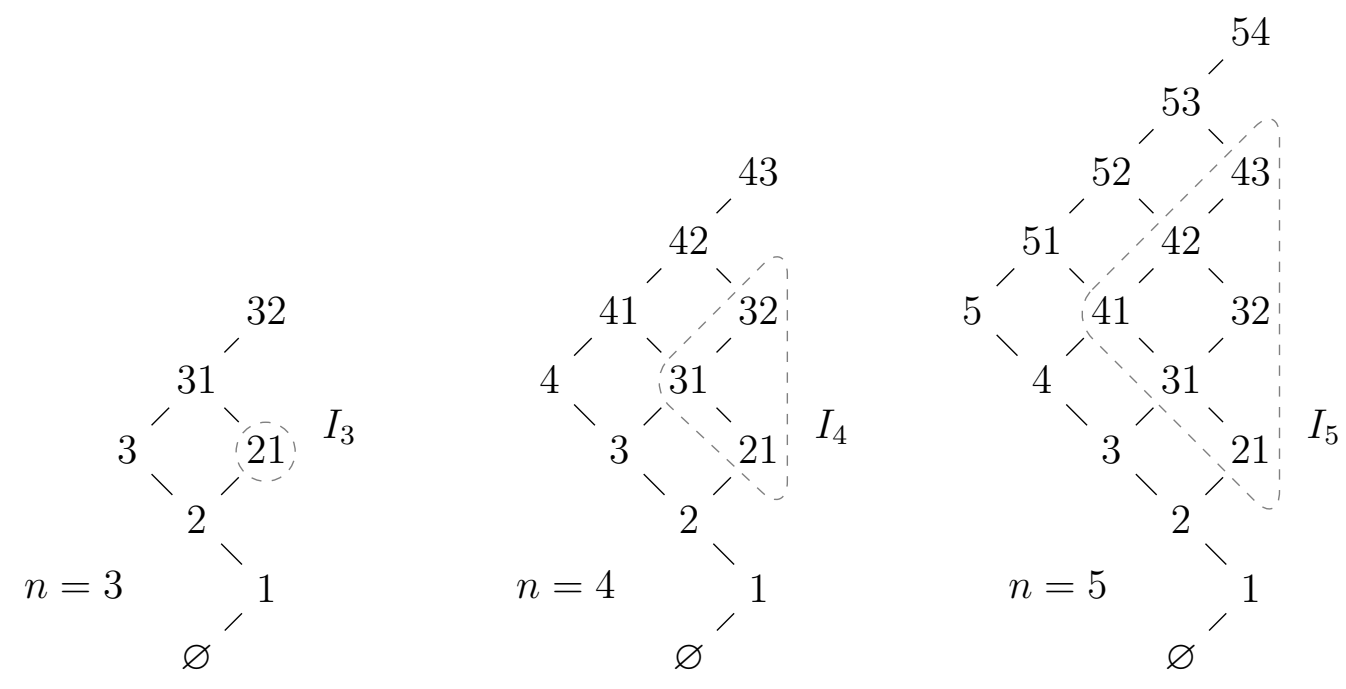

Figure 5: The lattice $F_{n, 2}$ induced by $1 \prec 2 \prec \cdots \prec n$ and de Finetti's condition for $n=3,4,5$. The set $I_{n}$ contains the doubletons whose comparison with the singleton $n$ is not determined by de Finetti's condition.

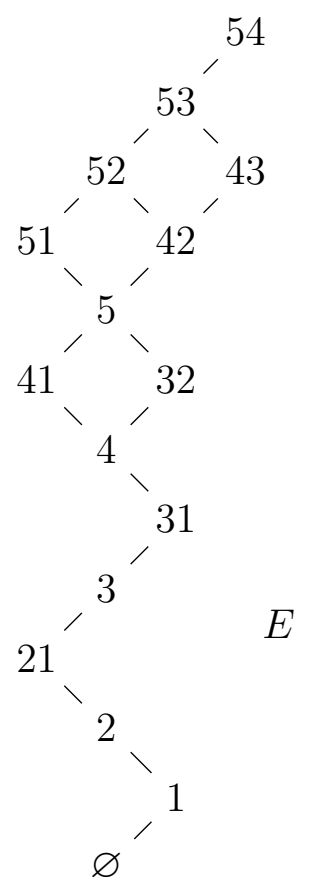

(a)

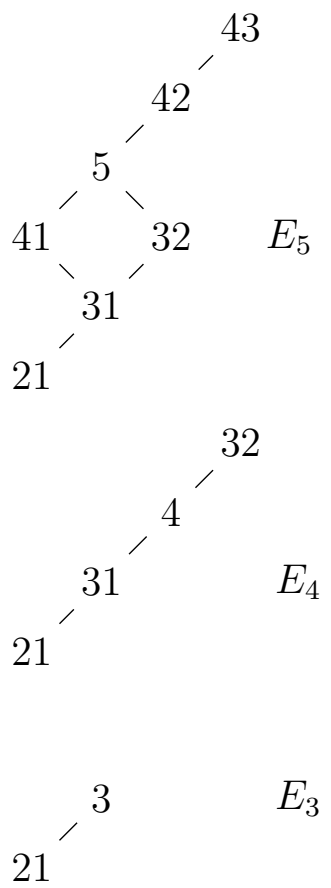

(b)

Figure 6: (a) A poset refinement $E \in \mathcal{F}_{5,2}^{1}$. Each singleton is comparable with every other set. (b) The subposets $E_{3}, E_{4}$ and $E_{5}$ of $E$. 
The column constraint (K2) of the kagog triangle will correspond to having singleton de Finetti refinements $\left(E_{3}, E_{4}, \ldots, E_{n}\right)$ whose union also abides by de Finetti's condition.

We begin by introducing a convenient $k$-list version of the power set $\mathcal{P}([k])$. Let

$$
\mathcal{L}([k])=\left\{(\underbrace{0, \ldots, 0}_{k-j}, s_{1}, s_{2}, \ldots, s_{j}): 0 \leqslant j \leqslant k \text { and } 1 \leqslant s_{1}<s_{2}<\cdots<s_{j} \leqslant k\right\}
$$

be the set of $k$-lists produced by listing the elements of $S \subset[k]$ in increasing order and then prepending $k-|S|$ zeros.

Lemma 14. Each row $1 \leqslant k \leqslant n-1$ of a kagog triangle in $\mathcal{K}_{n}$ is an element of $\mathcal{L}([k])$.

Proof. The constraint (K4) on row $k$ of a kagog triangle in $\mathcal{K}_{n}$ is identical to the conditions on a list in $\mathcal{L}([k])$.

Lemma 15. For $n \geqslant 3, \Phi\left(I_{n}\right)$ is in bijection with $\mathcal{L}([n-2])$.

Let us build some intuition with two examples. First, we consider refinements in $\Phi\left(I_{5}\right)$. We must determine the comparisons of the singleton $\{5\}$ with the doubletons in the lattice $I_{5}$. By interweaving empty boxes among the doubletons, we obtain the template

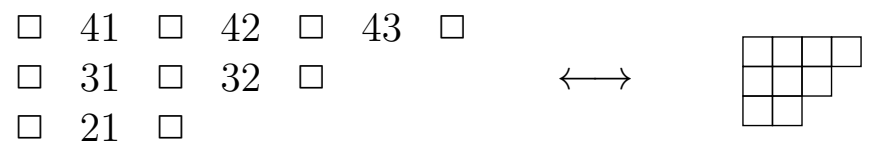

where omitting the doubletons gives the Ferrers diagram for the integer partition $(4,3,2)$. Specifying the comparisons with singleton $\{5\}$ is equivalent to placing a dot in each row of $(4,3,2)$. Looking only at the top row, placing a 5 in the first box

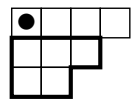

means that $5 \prec 41$. This puts no further de Finetti restrictions on the remaining two rows. The four ways to complete this configuration are
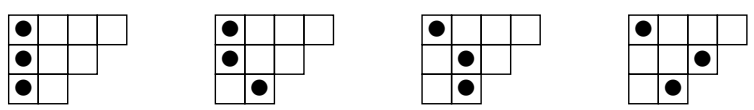

which correspond to the respective comparisons

$$
5 \prec 21 \quad 21 \prec 5 \prec 31 \quad 31 \prec 5 \prec 32 \quad 32 \prec 5 \prec 41 .
$$

Our final step is to count the boxes to the right of these dots, starting from the bottom row and moving up. This results in the lists $(1,2,3),(0,2,3),(0,1,3)$ and $(0,0,3)$ from $\mathcal{L}([3])$.

Next, we consider refinements in $\Phi\left(I_{7}\right)$. Specifying the comparisons of singleton $\{7\}$ with the doubletons in $I_{7}$ is equivalent to placing a dot in each row of the integer partition $(6,5,4,3,2)$. Suppose that we place a 7 in the third box of the first row, corresponding to $62 \prec 7 \prec 63$. Now de Finetti's condition leads to $21 \prec 32 \prec 42 \prec 52 \prec 62 \prec 7$, which yields the partially filled diagram 


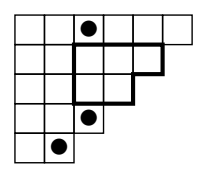

which contains a shifted copy of partition $(3,2)$ whose rows must each be assigned a dot. This can be done in four ways, and counting the boxes to the right of the dots gives the lists $(0,0,1,2,3),(0,0,0,2,3),(0,0,0,1,3)$ and $(0,0,0,0,3)$ from $\mathcal{L}([5])$. We now prove Lemma 15 by strong induction.

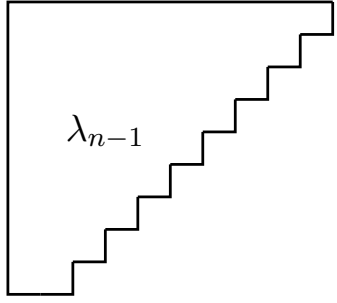

(a)

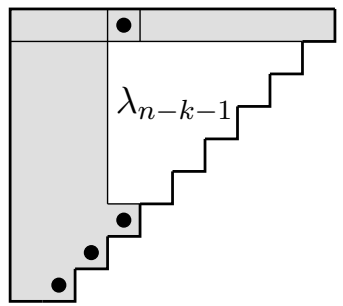

(c)

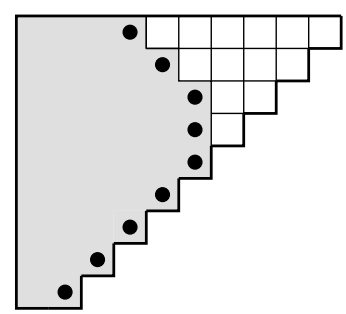

(c)

Figure 7: (a) The Ferrers diagram $\lambda_{n-1}=(n-1, n-2, \ldots, 2)$ for ordering singleton $\{n\}$ with the doubletons. (b) When $\{n-1, k-1\} \prec n \prec\{n-1, k\}$, we place a dot in the $k$ th position. This places de Finetti restrictions on the remaining rows. Completing the order is equivalent to choosing an order inside template $\lambda_{n-k-1}$. (c) Counting the boxes to the right of the dots gives the subset $\{6,4,2,1\} \subset[n-2]$.

Proof of Lemma 15. We recursively define the bijection $f: \Phi\left(I_{n}\right) \rightarrow \mathcal{L}([n-2])$. For $n=3$, we map the ordering with $21 \prec 3$ to the list (0) and the ordering with $3 \prec 21$ to the list (1). Assume that we have specified the bijection $f: \Phi\left(I_{\ell}\right) \rightarrow \mathcal{L}([\ell-2])$ for $2 \leqslant \ell<n$. We determine the image of a refinement $E \in \Phi\left(I_{n}\right)$. As in the above examples, we represent $E$ by placing a dot in each row of the Ferrers diagram $\lambda_{n-1}=(n-1, n-2, \ldots, 2)$, see Figure 7 (a). Our target list in $L \in \mathcal{L}([n-2])$ will be obtained by counting the boxes to the right of the dot in each row.

Placing a dot in position $1 \leqslant k \leqslant n-1$ of the first row of the template

$$
\square\{n-1,1\} \quad \square \quad\{n-1,2\} \quad \square \quad \cdots \quad \square \quad\{n-1, n-3\} \quad \square \quad\{n-1, n-2\}
$$

resolves the ordering of singleton $\{n\}$ with the doubletons $\{n-1, j\}$ for $1 \leqslant j \leqslant n-2$. If $k=1$ then $\{n\} \prec\{n-1,1\}$; if $1<k<n-1$ then $\{n-1, k-1\} \prec\{n\} \prec\{n-1, k\}$; and if $k=n-1$ then $\{n-1, n-2\} \prec\{n\}$. Condition (F2) puts constraints on the remaining rows. For rows $2 \leqslant i \leqslant n-k-1$, we must place $n$ in position $k$ or higher. For rows $i>n-k-1$, we must place $n$ in the rightmost (diagonal) position. Therefore, we can restrict our attention to rows $2 \leqslant i \leqslant n-k-1$ and positions $k \leqslant j \leqslant n-2$. But this is simply a translation of the mapping $f: \Phi\left(I_{n-k}\right) \rightarrow \mathcal{L}([n-k-2])$ via a copy of $\lambda_{n-k-1}$, 
see Figure $7(\mathrm{~b})$. Let $\left(a_{1}, a_{2}, \ldots, a_{n-k-2}\right) \in \mathcal{L}([n-k-2])$ be the image of this mapping. Noting that $a_{n-k-2} \leqslant n-k-2$, we set

$$
f(E)=(\underbrace{0, \ldots, 0}_{k}, a_{1}, a_{2}, \ldots, a_{n-k-2}, n-k-1) .
$$

The values in this list are the number of boxes to the right of the dots in Figure $7(\mathrm{c})$, when ordered from bottom to top.

We can now prove that the set of de Finetti refinements $\mathcal{F}_{n, 2}^{1}$ is in bijection with the set of kagog triangles $\mathcal{K}_{n-1}$.

Proof of Theorem 10. Let $E \in \mathcal{F}_{n, 2}^{1}$ be a de Finetti refinement of $F_{n, 2}$ so that every singleton is universally comparable in $E$. Consider $\left(E_{3}, E_{4}, \ldots, E_{n}\right)$ where $E_{k} \in \Phi\left(I_{k}\right)$ is the poset refinement of $I_{k} \cup\{k\}$ induced by $E$. Create a triangular array $T=T(i, j)$ for $1 \leqslant j \leqslant i \leqslant n-2$ by applying the mapping $f$ from Lemma 15 to each element in this list of refinements, using the indexing convention

$$
f\left(E_{k}\right)=(T(k-2,1), T(k-2,2), \cdots, T(k-2, k-2)), \quad 3 \leqslant k \leqslant n .
$$

By Lemma 15, each row satisfies the kagog row constraint. Meanwhile, the refinement $E$ satisfies de Finetti's condition (F2). In particular, for any $1 \leqslant i<j<k \leqslant n$, if $\{k\} \prec\{j, i\}$ then $\{k-1\} \prec\{j, i\}$. In terms of triangle $T$, this means that $T(k-2, n-j) \geqslant$ $T(k-3, n-j)$. For $2 \leqslant j \leqslant n-1$, this is precisely the contraint that column $n-k$ of a kagog triangle must be weakly decreasing constraint on column. Column $n-1$ has a single entry, so the final column is (vacuously) weakly increasing.

\subsection{The bijection from $\mathcal{M}_{n}$ to $\mathcal{K}_{n}$}

We now prove Theorem 12. Recall that each triangular family $\mathcal{T}_{n}$ forms a distributive lattice and that Definition 13 constructs two-color pyramids in relation to the maximum and minimum triangle of $\mathcal{T}_{n}$.

The minimum magog triangle has $M_{\min }(i, j)=1$ for every entry $(i, j)$ and the maximum magog triangle has $M_{\max }(i, j)=j$ for every entry $(i, j)$. Our first transformation is to subtract $M_{\min }$ from each magog triangle. The rightmost column becomes all-zero, so we omit it and reindex. This leads to the family of omagog triangles (short for "zeroedmagog" triangles).

Definition 16. An omagog triangle $M^{\circ}$ of index $n$ is an array of nonnegative integers $M^{\circ}(i, j)$ such that

(OM1) $1 \leqslant j \leqslant i \leqslant n-1$, so the array is triangular

(OM2) $M^{\circ}(i, j) \leqslant j$, so the entries in column $k$ are at most $\mathrm{j}$;

(OM3) $M^{\circ}(i, j) \leqslant M^{\circ}(i+1, j)$, so columns are weakly increasing; and 
(OM4) $M^{\circ}(i, j) \leqslant M^{\circ}(i, j+1)$, so rows are weakly increasing.

We use $\mathcal{M}_{n}^{\circ}$ to denote the set of all omagog triangles of index $n$.

The set $\mathcal{M}_{3}^{\circ}$ appears in Figure 8, with elements ordered so that they biject to the magog triangles of (3). The minimum omagog triangle satisfies $M_{\min }^{\circ}(i, j)=0$ and the maximum omagog triangle satisfies $M_{\max }^{\circ}(i, j)=j$ for all entries $(i, j)$.

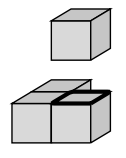

0

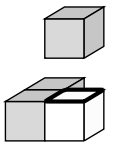

0

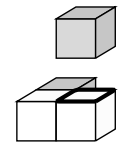

0

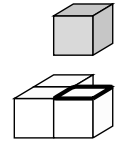

1

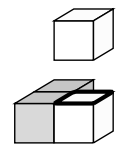

0

$\begin{array}{llll}0 & 0 & 0 & 1\end{array}$

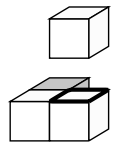

0

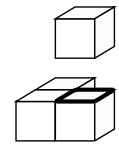

1

12

Figure 8: The omagog triangles $\mathcal{M}_{3}^{\circ}$ and the corresponding two-color omagog pyramids $\triangle \mathcal{M}_{3}^{\circ}$, drawn using a right-handed coordinate system. The pyramids are sliced into horizontal layers. The shadow of a layer is thickly drawn on the layer below.

Proof of Theorem 12. We create a bijection $\psi$ from omagog pyramids $\triangle \mathcal{M}_{n}^{\circ}$ to kagog pyramids $\triangle \mathcal{K}_{n}$ via a sequence of elementary transformations.

Recall that a two-color pyramid $\triangle T$ is a collection of cubes $(i, j, k)$ that are colored white or gray. Renaming these colors as color 1 and color 0 , respectively, then the twocolor pyramid becomes a binary function on the set of admissible coordinates, that is $\triangle M^{\circ}:(i, j, k) \mapsto\{0,1\}$. Viewing $\triangle M^{\circ}$ as a function allows us to describe the collection $\triangle \mathcal{M}_{n}^{\circ}$ of two-color pyramids with a system of inequalities. We have

- $\triangle M^{\circ}(i, j, k)$ is defined for $1 \leqslant k \leqslant j \leqslant i \leqslant n-1$.

- $\triangle M^{\circ}(i, j, k) \leqslant \triangle M^{\circ}(i+1, j, k)$ : the columns of the magog triangle are nondecreasing,

- $\triangle M^{\circ}(i, j, k) \leqslant \triangle M^{\circ}(i, j+1, k)$ : the rows of the magog triangle are nondecreasing, and

- $\triangle M^{\circ}(i, j, k+1) \leqslant \triangle M^{\circ}(i, j, k)$ : color 1 (white, present) cubes are below color 0 (gray, absent) cubes, so the cubes that are present obey "gravity."

We now perform our four step transformation $\psi$. We use a right-handed coordinate system, so that the positive $x$-axis points out of the paper (southward) and the negative $x$-axis points into the paper (northward). The $y$-axis and $z$-axis lie in the plane of the paper, corresponding to cardinal east/west and up/down directions, respectively.

- Step 1: Invert the colors, or exchange color 0 for color 1 and vice versa. This reverses the inequalities. 
- Step 2: Push all cubes north in their respective column so that row 1 has length $n-1$. This is equivalent to moving the cube $(i, j, k)$ to $(i-(j-1), j, k)$.

- Step 3: Tip the entire stack over the $y$-axis via a clockwise rotation by $\pi / 2$ and then translate by $(n, 0,0)$. This is equivalent to moving the cube $(i, j, k)$ to $(n-k, j, i)$.

- Step 4: Reflect the stack through the plane $y=(n+1) / 2$. This is equivalent to moving the cube $(i, j, k)$ to $(i, n-j, k)$.

After composing these four steps, cube $(i, j, k)$ switches color and moves to $(n-k, n-$ $j, i-j+1)$. Figure 9 shows the mapping $\psi$ for an omagog pyramid in $\triangle \mathcal{M}_{4}^{\circ}$.

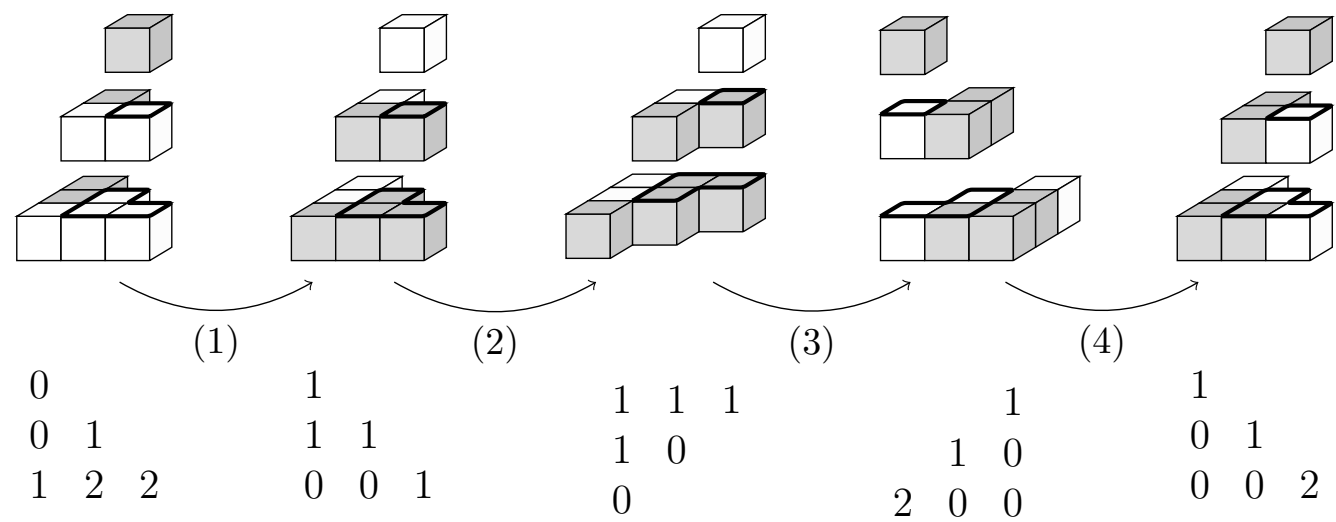

Figure 9: Example of the omagog to kagog bijection $\psi$. The pyramids are drawn using a right-handed coordinate system with the positive $x$-axis pointing out of the page. (1) Invert the colors. (2) push the cubes northward along the columns. (3) Tip the stack around the $y$-axis and then translate by $(n, 0,0)$. (4) Reflect through the plane $y=$ $(n+1) / 2$.

Updating the omagog pyramid inequalities at every step leads to the following algebraic constraints for some pyramid $\triangle P$ :

(P1) $\triangle P(i, j, k)$ is defined for $1 \leqslant k \leqslant j \leqslant i \leqslant n-1$.

(P2) $\triangle P(i, j, k) \geqslant \triangle P(i+1, j, k)$,

(P3) $\triangle P(i, j, k) \geqslant \triangle P(i, j-1, k-1)$, and

(P4) $\triangle P(i, j, k) \geqslant \triangle P(i, j, k+1)$.

These pyramid inequalities correspond to the kagog triangle constraints of Definition 9 , where we must recall that color 1 (white) cubes are present and color 0 (gray) cubes are absent. Condition (P1) ensures that the domain for admissible cubes $(i, j, k)$ is correct and that the height of tower $(i, j)$ is at most $j$, so (K1) and (K2) hold. Condition (P4) states that the cubes adhere to gravity: color 1 blocks must appear below color 0 blocks. Conditions (P2) and (P4) ensure that the columns are weakly decreasing, so (K3) holds. 
Conditions (P3) and (P4) ensure that the rows are strictly increasing after the first nonzero entry, so (K4) holds. Indeed, if cube $(i, j-1, k-1)$ is color 1 , then $(i, j, k)$ is color 1 , so the tower at $(i, j)$ must be taller than the tower at $(i, j-1)$.

\subsection{A Catalan Submapping}

In this brief digression, we show that the mapping $\psi: \triangle \mathcal{M}_{n}^{\circ} \rightarrow \triangle \mathcal{K}_{n}$ induces a natural bijection between Catalan subfamilies of these pyramids. We start by describing two known Catalan families [31]. Let $\mathcal{S}_{n}$ denote the set of nondecreasing sequences $\left(s_{0}, s_{1}, \ldots, s_{n-1}\right)$ where $0 \leqslant s_{i} \leqslant i$ for $0 \leqslant i \leqslant n-1$ and $s_{i} \leqslant s_{i+1}$ for $0 \leqslant i \leqslant n-2$. Let $\mathcal{C}_{n}$ denote the set of coin pyramids whose bottom row contains $n$ consecutive coins and such that every additional coin is above and between two other coins. Both families are shown in Figure 10

Next, we define our associated pyramid families. Let $\mathcal{S}_{n}^{\prime} \subset \mathcal{M}_{n}^{\circ}$ be the set of omagog triangles whose first $n-2$ rows are all zero. Let $\mathcal{C}_{n}^{\prime} \subset \mathcal{K}_{n}$ be the set of kagog triangles such that for $1 \leqslant j \leqslant n-1$, every entry in column $j$ is either $j-1$ or $j$.

Proposition 17. Let $\mathcal{S}_{n}, \mathcal{C}_{n}, \mathcal{S}_{n}^{\prime}$ and $\mathcal{C}_{n}^{\prime}$ be the families defined above.

(a) There is an elementary bijection $\sigma: \mathcal{S}_{n} \rightarrow \mathcal{C}_{n}$.

(b) There is an elementary bijection $\rho: \mathcal{S}_{n} \rightarrow \mathcal{S}_{n}^{\prime}$.

(c) There is an elementary bijection $\tau: \mathcal{C}_{n} \rightarrow \mathcal{C}_{n}^{\prime}$.

(d) Restricting the bijection $\psi: \triangle \mathcal{M}_{n}^{\circ} \rightarrow \triangle \mathcal{K}_{n}$ from Theorem 12 to $\triangle \mathcal{S}_{n}^{\prime}$ gives a bijection to $\triangle \mathcal{C}_{n}^{\prime}$. Furthermore, this bijection has a natural interpretation in terms of monotone sequences and coin pyramids. Namely, $\sigma=\tau^{-1} \circ \psi \circ \rho$.

Proof. Figure 10 shows the families $\mathcal{S}_{3}, \mathcal{C}_{3}, \mathcal{S}_{3}^{\prime}, \mathcal{C}_{3}^{\prime}$. It also shows two families $\mathcal{H}_{3}, \mathcal{H}_{3}^{\prime}$ of hybrid configurations that are essential in multiple stages of the proof.

Proof of (a). Our bijection relates $\mathcal{S}_{n}$ to $\mathcal{C}_{n}$ via the set $\mathcal{P}_{n}$ of lattice paths from $(0,0)$ to $(n, n)$ that never travel above the diagonal $y=x$, composing mappings described in [31]. First, we map sequence $s=\left(s_{0}, s_{1}, \ldots, s_{n-1}\right) \in \mathcal{S}_{n}$ to the lattice path $p \in \mathcal{P}_{n}$ whose $k$ th horizontal step is at height $s_{k}$. Next, we place gray (missing) coins in each square below $p$, and place white coins in each square above the path $p$, up to and including the squares along the diagonal $y=x$. Let $\mathcal{H}_{n}$ denote the hybrid family of configurations of paths and coins, where missing coins are gray. To complete the mapping $\sigma$, reflect the white coins in the hybrid configuration through $\theta=\pi / 8$ to obtain the corresponding coin pyramid (equivalently, we could rotate by $3 \pi / 4$ and then reflect across the $y$-axis).

Proof of (b). The monotone sequences $\mathcal{S}_{n}$ map quite simply to $\mathcal{S}_{n}^{\prime}$. The sequence $s \in \mathcal{S}_{n}$ maps to the omagog triangle in $\mathcal{M}_{n}^{\circ}$ whose final row is $\left(s_{1}, \ldots, s_{n-1}\right)$, and whose other rows are all-zero. This mapping is clearly a bijection.

Proof of (c). We map coin pyramids $\mathcal{C}_{n}$ to the triangles in $\mathcal{C}_{n}^{\prime}$ via the hybrid configurations in $\mathcal{H}_{n}$. After mapping a coin pyramid to its hybrid configuration in $\mathcal{H}_{n}$, we ignore the white coins on the diagonal (which correspond to the fixed base of the coin pyramid), 

$\begin{array}{lll}\mathcal{S}_{3}^{\prime} & 0 & \\ & 0 & 0\end{array}$
0
$\begin{array}{ll}0 & 1\end{array}$
0
$\begin{array}{ll}1 & 1\end{array}$
$\begin{array}{ll}0 & \\ 0 & 2\end{array}$
0
$\mathcal{S}_{3} \quad(0,0,0)$
$(0,0,1)$
$(0,1,1)$
$(0,0,2)$
$(0,1,2)$
$\mathcal{C}_{3}$
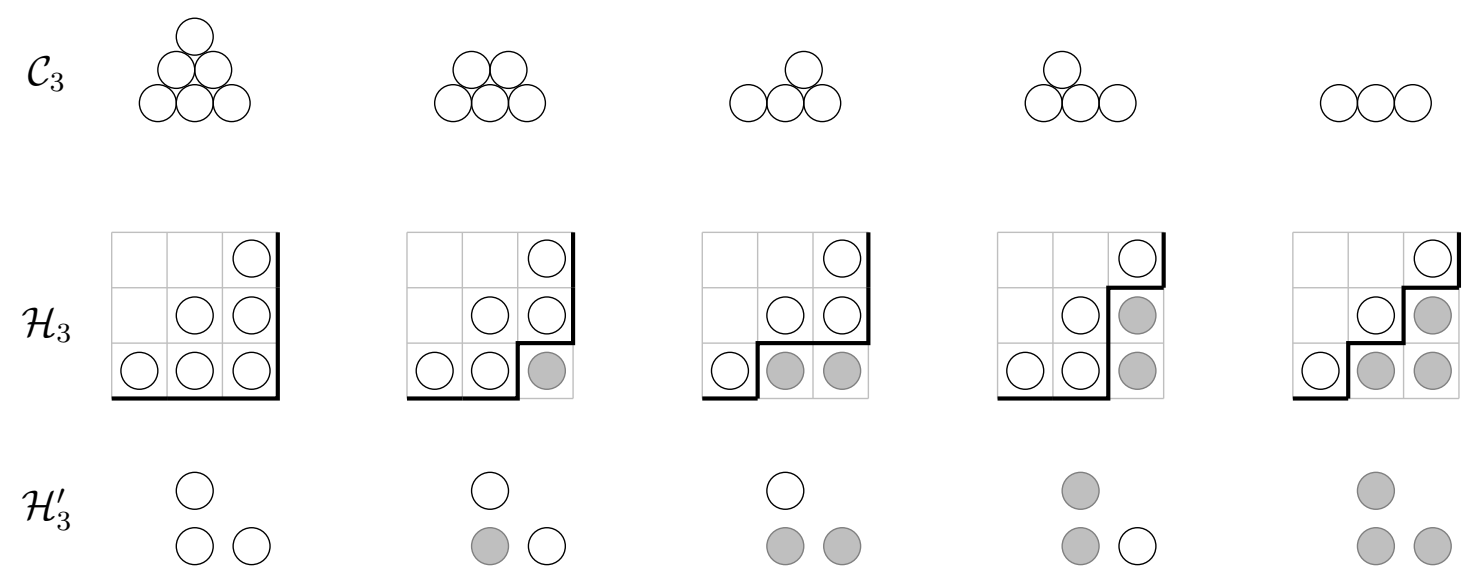
$\begin{array}{lll}\mathcal{C}_{3}^{\prime} & 1 & \\ & 1 & 2\end{array}$
$\begin{array}{ll}1 & \\ 0 & 2\end{array}$
1
$\begin{array}{ll}0 & 1\end{array}$
$\begin{array}{ll}0 & \\ 0 & 2\end{array}$
$\begin{array}{ll}0 & 1\end{array}$

Figure 10: Six Catalan families used in the proof of Proposition 17. The family $\mathcal{S}_{3}$ of monotone sequences $\left(s_{0}, s_{1}, s_{2}\right)$ maps simply to the subfamily $\mathcal{S}_{3}^{\prime}$ of omagog pyramids whose first row is zero. The family $\mathcal{C}_{3}$ of coin pyramids is in bijection with $\mathcal{S}_{3}$ via the hybrid family $\mathcal{H}_{3}$ consisting of lattice paths and coins, where $s_{k}$ is the height of the horizontal step starting at $x=k$. We map $\mathcal{C}_{3}$ to the subfamily $\mathcal{C}_{3}^{\prime}$ of kagog pyramids via family $\mathcal{H}_{3}^{\prime}$, the mirror image of the non-diagonal coins of $\mathcal{H}_{3}$.

and reflect the remaining coins across the vertical axis to get a triangular array of the appropriate shape. Let $\mathcal{H}_{n}^{\prime}$ denote the resulting family of triangular arrays of two-colored coins. Replace each white coin with a 1 and each gray coin with a 0 . Finally, add $j-1$ to the entries in column $j$ for $1 \leqslant j \leqslant n-1$. The result is a kagog triangle in $\mathcal{C}_{n}^{\prime}$. This invertible mapping is a bijection.

Proof of (d). First, we show that the bijection $\psi: \triangle \mathcal{M}_{n}^{\circ} \rightarrow \triangle \mathcal{K}_{n}$ maps $\triangle \mathcal{S}_{n}^{\prime}$ to $\triangle \mathcal{C}_{n}^{\prime}$. All of the white (present) blocks of $\triangle S \in \triangle \mathcal{S}_{n}^{\prime}$ are in row $n-1$. Let $\triangle K=\psi(\triangle S)$ where $\psi$ is the mapping in the proof of Theorem 12. Recall that in this mapping, the block $\triangle S(i, j, k)$ flips colors and moves to $\triangle K(n-k, n-j, i-j+1)$. In particular, the gray block $\triangle S(n-2, j, k)$ maps to the white block $\triangle K(n-k, n-j, n-j-1)$. This proves that every tower in column $\ell=n-j$ has height at least $\ell-1=n-j-1$; in other words, $\psi$ bijects $\triangle \mathcal{S}_{n}^{\prime}$ to $\triangle \mathcal{C}_{n}^{\prime}$.

It remains to show that the mapping $\psi$ corresponds to the mapping $\sigma: \mathcal{S}_{n} \rightarrow \mathcal{C}_{n}$. The key is to take a bird's eye view of a kagog pyramid $\triangle K \in \Delta \mathcal{C}_{n}^{\prime}$. This view only shows the topmost blocks; this is sufficient, since the blocks in the lower layers are all 
white. We will see that the coin colors of $h^{\prime} \in \mathcal{H}_{n}^{\prime}$ correspond to the block colors of the top layer of a unique $\triangle K=\psi(\triangle S)$. Keeping this intuition in mind, we conclude the proof. After mapping, the block $\triangle S(n-1, j, k)$ flips color and maps to the top-layer block $\triangle K(n-k, n-j, n-j)$. Suppose that $\triangle S(n-1, j, k)$ is white for $1 \leqslant k \leqslant \ell$ and gray for $\ell+1 \leqslant k \leqslant j$. This means that $\triangle K(n-k, n-j, n-j)$ is gray for $1 \leqslant k \leqslant \ell$ and white for $\ell+1 \leqslant k \leqslant j$. In other words, $\triangle K\left(k^{\prime}, j^{\prime}, j^{\prime}\right)$ is gray for $n-\ell \leqslant k^{\prime} \leqslant n-1$ and white for $j^{\prime} \leqslant k^{\prime} \leqslant n-\ell-1$. The bird's eye view of the pyramids of $\triangle \mathcal{K}_{n}$ bijects to the hybrid configurations of $\mathcal{H}_{n}^{\prime}$, where we replace the blocks with coins.

\section{The Gog Triangle Involution}

Given the success of the two-color cube pyramid view of magog triangles, we investigate two-color cube pyramids of gog triangles. Inspired by the mapping from omagog pyramids to kagog pyramids from 3.3, apply a similar transformation to gog triangles. This results in a natural involution on gog pyramids that corresponds to row reversal of the equivalent ASM. This section culminates in our proof of Theorem 6 .

Definition 18. A gog triangle $G$ of size $n$ is a triangular array of positive integers $G(i, j)$ such that

(G1) $1 \leqslant j \leqslant i \leqslant n$, so the array is triangular;

(G2) $1 \leqslant G(i, j) \leqslant n-i+j$, so entry $j$ in row $i$ is at most $n-i+j$;

(G3) $G(i, j)<G(i, j+1)$, so rows are strictly increasing;

(G4) $G(i, j) \geqslant G(i+1, j)$, so columns are weakly decreasing; and

(G5) $G(i, j) \leqslant G(i+1, j+1)$, so diagonals are weakly increasing.

We use $\mathcal{G}_{n}$ to denote the set of gog triangles of size $n$.

Note that we use (G2) in place of the standard condition that $G(n, j)=j$ for $1 \leqslant$ $j \leqslant n$; an elementary argument shows that they are interchangeable. Condition (G2), which applies to all rows of the triangle, is better suited for our two-colored cube pyramid argument.

The gog triangles of $\mathcal{G}_{3}$ are

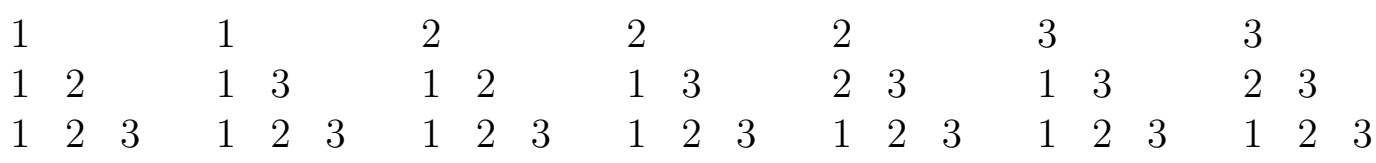

where once again, we have chosen to left-justify the triangular arrays.

Gog triangles (also known as monotone triangles) are in bijection with alternating sign matrices. An ASM is a $n \times n$ matrix of 0's, 1's and -1 's such that each or column sums 
to 1 , and the nonzero entries in each row or column alternate in sign. The seven $3 \times 3$ ASMs are

$$
\left[\begin{array}{lll}
1 & 0 & 0 \\
0 & 1 & 0 \\
0 & 0 & 1
\end{array}\right]\left[\begin{array}{lll}
1 & 0 & 0 \\
0 & 0 & 1 \\
0 & 1 & 0
\end{array}\right]\left[\begin{array}{lll}
0 & 1 & 0 \\
1 & 0 & 0 \\
0 & 0 & 1
\end{array}\right]\left[\begin{array}{ccc}
0 & 1 & 0 \\
1 & -1 & 1 \\
0 & 1 & 0
\end{array}\right]\left[\begin{array}{lll}
0 & 1 & 0 \\
0 & 0 & 1 \\
1 & 0 & 0
\end{array}\right]\left[\begin{array}{lll}
0 & 0 & 1 \\
1 & 0 & 0 \\
0 & 1 & 0
\end{array}\right]\left[\begin{array}{lll}
0 & 0 & 1 \\
0 & 1 & 0 \\
1 & 0 & 0
\end{array}\right] .
$$

We have listed the seven gog triangles in (5) in the same order as their corresponding $3 \times 3$ ASMs in (6). The bijective mapping of Mills et al. [24] between ASMs and gog triangles is simple to describe: the $j$ th row of the gog triangle records the locations of the 1 's in the vector obtained by adding the first $j$ rows of the corresponding ASM.

We now prove Theorem 6: there is a natural gog triangle involution $f: \mathcal{G}_{n} \rightarrow \mathcal{G}_{n}$ that corresponds to both (1) an affine transformation of two-color pyramids, and (2) reversing the order of the rows of the corresponding ASM.

Analogous to Section 3.3, we start by defining ogog triangles. The minimum gog triangle has $G_{\min }(i, j)=j$ for all entries $(i, j)$, while the maximum gog triangle has $G_{\max }(i, j)=n-i+j$. For every gog triangle, we construct its ogog counterpart by subtracting the minimum gog triangle. The last row in every gog triangle is always $\left[\begin{array}{llll}1 & 2 & \cdots & n\end{array}\right]$ since it has length $n$ and is strictly increasing. As such, every ogog triangle has a final row of zeros, which we omit from ogog triangle.

Definition 19. An ogog triangle $G^{\circ}$ of index $n$ is a triangular array of nonnegative integers $G^{\circ}(i, j)$ such that

(OG1) $1 \leqslant j \leqslant i \leqslant n-1$, so the array is triangular;

(OG2) $0 \leqslant G^{\circ}(i, j) \leqslant n-i$, so values in row $i$ are at most $n-i$;

(OG3) $G^{\circ}(i, j) \leqslant G^{\circ}(i, j+1)$, so rows are weakly increasing;

(OG4) $G^{\circ}(i, j) \geqslant G^{\circ}(i+1, j)$, so columns are weakly decreasing; and

(OG5) $G^{\circ}(i, j) \leqslant G^{\circ}(i+1, j+1)+1$, so diagonals cannot decrease by more than 1 .

We use $\mathcal{G}_{n}^{\circ}$ to denote the set of all ogog triangles of index $n$.

For example, the ogog triangles of $\mathcal{G}_{3}^{\circ}$ are

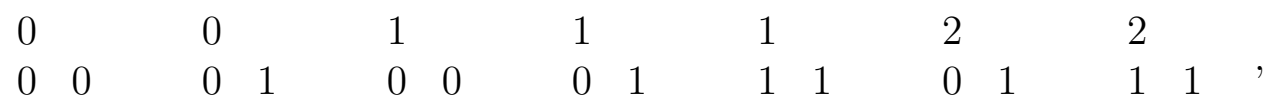

where these ogog triangles are ordered so that they biject to the gog triangles in (5). As constructed, the color 1 (white) cubes are present in the ogog triangle, while the color 0 (gray) cubes are absent. Our next lemma states that the gray cubes also represent a gog triangle.

Lemma 20. Let $G^{\circ}$ be an ogog triangle and let $\triangle G^{\circ}$ be its two-color cube pyramid representation. The color 0 cubes of $\triangle G^{\circ}$ are an affine transformation of another ogog triangle. 
Note that this correspondence is an involution on the set of ogog triangles: swapping the colors twice leads us back to the original two-coloring of the cube pyramid.

Proof. Similar to the proof of Lemma 12, we describe ogog pyramids via a set of inequalities, perform a multistep transformation and then check that the resulting inequalities also describe the set of ogog pyramids. The inequalities for ogog pyramids are:

- $\triangle G^{\circ}(i, j, k)$ is defined $1 \leqslant j \leqslant i \leqslant n-1$ : length of row $i$ is at most $i$,

- $\triangle G^{\circ}(i, j, k)$ is defined $1 \leqslant k \leqslant n-i$ : height of row $i$ is at most $n-i$,

- if $j<i$, then $\triangle G^{\circ}(i, j+1, k) \geqslant \triangle G^{\circ}(i, j, k)$ : rows are weakly increasing,

- if $i>1$, then $\triangle G^{\circ}(i-1, j, k) \geqslant \triangle G^{\circ}(i, j, k)$ : columns are weakly decreasing

- if $i<n$, then $\triangle G^{\circ}(i+1, j+1, k-1) \geqslant \triangle G^{\circ}(i, j, k)$ : diagonals cannot decrease by more than 1 , and

- if $k>1$, then $\triangle G^{\circ}(i, j, k-1) \geqslant \triangle G^{\circ}(i, j, k)$ : the present cubes obey gravity.

The three-step mapping $\varphi$ is:

- Step One: Invert the colors, or exchange color 1 for color 0 and vice versa. This reverses the inequalities.

- Step Two: Perform a quarter rotation of $\mathbb{R}^{3}$ about the $x$-axis. This moves the cube $(i, j, k)$ to position $(i,-k, j)$. This tips the two-color cube pyramid onto its side.

- Step Three: Rotate by $\pi$ around the $z$-axis and then translate by $(n, 0,0)$. This moves cube $(i, j, k)$ to $(n-i,-j, k)$.

After composing these three steps, cube $(i, j, k)$ switches color and moves to $(n-i, k, j)$. Figure 11 exemplifies the mapping $\varphi$ for an ogog pyramid from $\triangle \mathcal{G}_{4}^{\circ}$.

Careful algebra shows that the resulting constraints are a permutation of the algebraic inequalities for an ogog cube pyramid. As such, this mapping takes one gog triangle to another gog triangle. This affine mapping is an involution, so it is bijective.

The ogog pyramids are in bijection with gog triangles, and hence also in bijection with alternating sign matrices. Our next Theorem shows that the involution $\varphi$ of Lemma 20 reverses the rows of the associated ASM.

Theorem 21. Let $\varphi$ be the ogog pyramid involution of Lemma 20. Let $A$ be an $n \times n$ alternating sign matrix corresponding to ogog triangle $G^{\circ}$ with two-color cube pyramid $\triangle G^{\circ}$. Let $\triangle H^{\circ}=\varphi\left(\triangle G^{\circ}\right)$ and let $H^{\circ}$ be the associated ogog triangle. Then $H^{\circ}$ is the ogog triangle corresponding to processing the rows of $A$ in reverse order.

Figure 12 shows an example of how the alternating sign matrices $A$ and $B$ corresponding to ogog pyramids $\triangle G^{\circ}$ and $\triangle H^{\circ}=\varphi\left(\triangle G^{\circ}\right)$ are the row reversals of one another. 


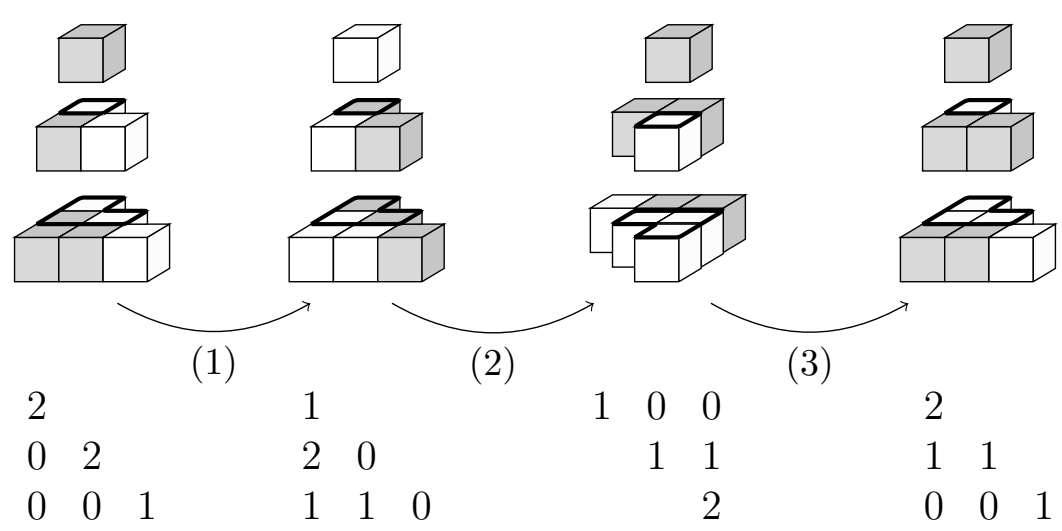

Figure 11: Example of the ogog to ogog bijection $\varphi$. The pyramids use a right-handed coordinate system in which the postive $x$-axis points out of the paper. (1) Invert the colors. (2) Tip the stack around the $x$-axis by a quarter turn. (3) Rotate one half turn about the $z$-axis, then translate by $(n, 0,0)$.

Proof. Starting with the alternating sign matrix $A$, we obtain the ogog triangle $G^{\circ}$ as follows. First, we create the matrix $A^{\prime}$ whose $i$ th row is the sum of the first $i$ rows of $A$. This is a $0-1$ matrix whose $i$ th row contains exactly $i$ ones. We convert $A^{\prime}$ into a gog triangle $G$ by reporting the indices of the ones in each row. We then set $G^{\circ}=G-G_{\min }$, which corresponds to subtracting $[1,2, \ldots, i]$ from row $i$ of $G$ for $1 \leqslant i \leqslant n$ and then deleting the final row (which is all-zero).

Let $A_{i}$ denote the $i$ th row of $A$ and let $A_{i}^{\prime}=\sum_{k=1}^{k} A_{i}$ denote the $i$ th row of the partial sum matrix $A^{\prime}$. Let $1 \leqslant a_{1}^{\prime}<a_{2}^{\prime}<\cdots<a_{i}^{\prime} \leqslant n$ denote the locations of the ones in row $A_{i}^{\prime}$. Then $G(i, j)=a_{j}^{\prime}$, or equivalently $\left[a_{1}^{\prime}, a_{2}^{\prime}, \cdots, a_{i}^{\prime}\right]$ is the $i$ th row of the gog triangle $G$. The entries satisfy

$$
\begin{aligned}
1 & \leqslant a_{1}^{\prime} \leqslant n-i+1 \\
a_{j-1}^{\prime} & <a_{j}^{\prime} \leqslant n-i+j, \quad 2 \leqslant j \leqslant i .
\end{aligned}
$$

Row $i$ of ogog triangle $G^{\circ}$ is

$$
\left[a_{1}^{\prime}-1, a_{2}^{\prime}-2 \cdots, a_{i}^{\prime}-i\right] .
$$

We start with row $n-1$ of our triangle, as it is the simplest row to comprehend. Row $n-1$ of gog triangle $G$ is $\left[a_{1}^{\prime}, a_{2}^{\prime}, \ldots, a_{n-1}^{\prime}\right]$, which is missing a single number $\ell \in[n]$, namely the location $\ell$ of the unique one in row $n$ of $A$. By (7), the corresponding ogog row consists of $\ell-1$ zeros followed by $n-\ell$ ones.

Consider this row in the context of the two-color ogog pyramid $\triangle G^{\circ}$ and its image $\triangle H^{\circ}=\varphi\left(\triangle G^{\circ}\right)$. Row $n-1$ of pyramid $\triangle G^{\circ}$ has height 1 . It contains $\ell-1$ cubes of color 0 , followed by $n-\ell$ cubes of color 1 . After transformation $\varphi$, the cube $(n-1, j, 1)$ switches color and moves to $(1,1, j)$. So $\triangle H^{\circ}$ has a tower of blocks at $(1,1)$ of height $n-1$, with $\ell-1$ cubes of color 1 below $n-\ell$ cubes of color 0 . It follows that ogog triangle $H^{\circ}$ has $H^{\circ}(1,1)=\ell-1$, and thus the corresponding gog triangle $H$ has $H(1,1)=\ell$. This 


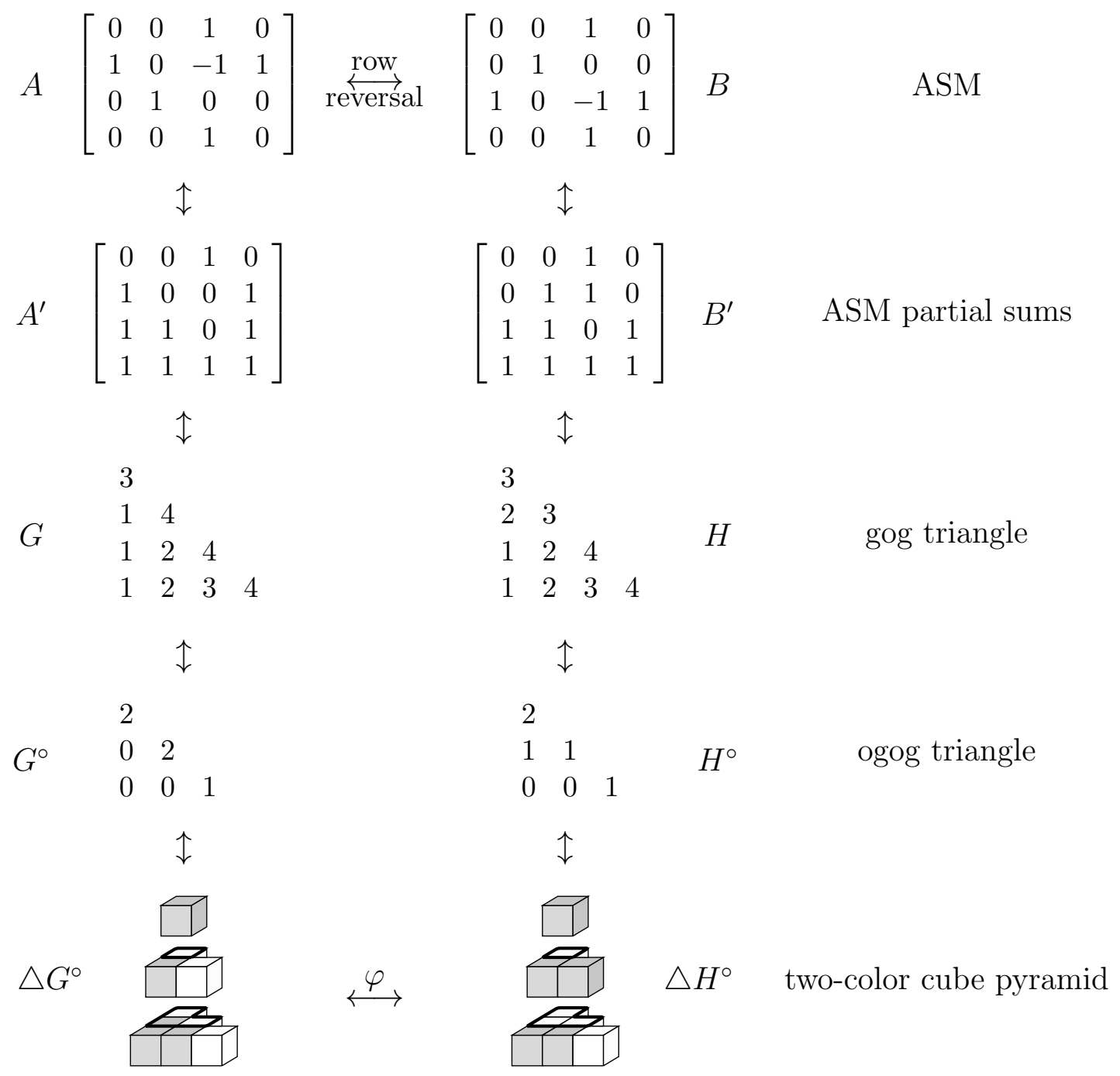

Figure 12: The alternating sign matrices $A$ and $B$ corresponding to the two-color cube pyramids $\triangle G^{\circ}$ and $\triangle H^{\circ}=\varphi\left(\triangle G^{\circ}\right)$ are the row reversals of one another.

confirms that the first row of gog triangle $H$ corresponds to the last row of matrix $A$, as desired.

We now handle a generic row $i$ of ogog triangle $G^{\circ}$; Figure 13 shows an example. The entries of row $i$ are a weakly increasing list of length $i$, drawn from $\{0,1, \ldots, n-i\}$. Let $0 \leqslant s_{m} \leqslant i$ be the number of consecutive $m$ 's in this list, so that

$$
\sum_{m=0}^{n-i} s_{m}=i .
$$


In the corresponding gog triangle $G$, row $i$ is missing the integers

$$
1+p+\sum_{k=0}^{p} s_{k} \quad \text { where } \quad 0 \leqslant p \leqslant n-i-1 .
$$

Let us pause to make some key observations. The missing integers in row $i$ of $G$ are precisely the locations of the zeros in the partial sum $A_{i}^{\prime}=\sum_{\ell=1}^{i} A_{\ell}$. Since the sum of all the rows yields the all-ones vector, these are also the locations of the ones in the partial sum $\sum_{\ell=i+1}^{n} A_{\ell}$. Of course, summing the last $n-i$ rows of $A$ is the same as summing the first $n-i$ rows of the row reversal of $A$.

Next, we translate our observations into statements about two-color pyramids. When we convert ogog triangle $G^{\circ}$ into pyramid $\triangle G^{\circ}$, row $i$ of $G^{\circ}$ maps to the $i \times(n-i)$ wall of cubes

$$
\triangle G_{i}^{\circ}=\{(i, j, k): 1 \leqslant j \leqslant i \text { and } 1 \leqslant k \leqslant n-i\} .
$$

The layer of wall $\triangle G_{i}^{\circ}$ at height $k$ consists of $\sum_{m=0}^{k-1} s_{m}$ cubes of color 0 followed by $\sum_{m=k}^{n-i} s_{m}$ cubes of color 1 . The transformation $\varphi: \triangle G^{\circ} \mapsto \triangle H^{\circ}$ maps $\triangle G_{i}^{\circ}$ to the $(n-i) \times i$ wall

$$
\triangle H_{n-i}^{\circ}=\{(n-i, k, j): 1 \leqslant k \leqslant n-i \text { and } 1 \leqslant j \leqslant i\} .
$$

We have inverted the colors and exchanged vertical and horizontal, so the tower of wall $\triangle H_{n-i}^{\circ}$ at $(n-i, k)$ consists of $\sum_{m=0}^{k-1} s_{m}$ cubes of color 1 , stacked below $\sum_{m=k}^{n-i} s_{m}$ cubes of color 0 .

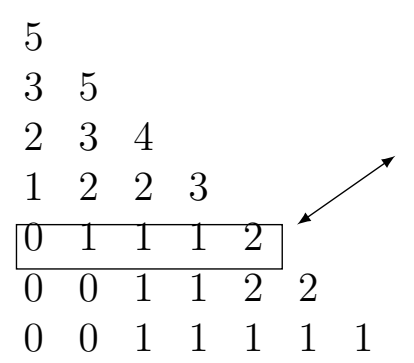

$G^{\circ}$

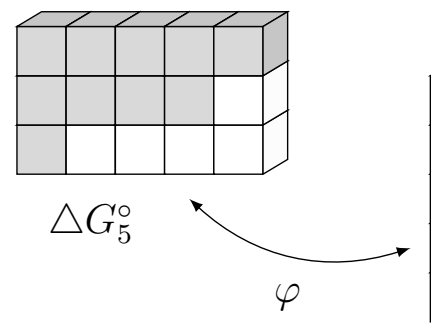

$\triangle H_{3}^{\circ}$

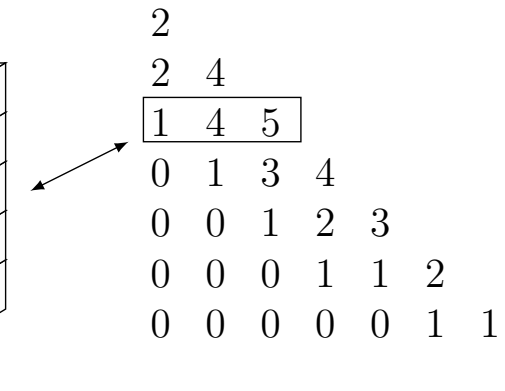

$H^{\circ}$

Figure 13: An ogog triangle $G^{\circ}$ from $\mathcal{G}_{8}^{\circ}$ and its image $H^{\circ}$ via the invertible mapping $\varphi$. Row 5 of triangle $G^{\circ}$ becomes pyramid wall $\triangle G_{5}^{\circ}$ which maps via $\varphi$ to pyramid wall $\triangle H_{3}^{\circ}$ and then to row 3 of triangle $H^{\circ}$.

We now translate the structure of pyramid $\triangle H^{\circ}$ into the triangle setting. Ogog triangle $H^{\circ}$ has $H^{\circ}(n-i, k)=\sum_{m=0}^{k-1} s_{m}$ for $1 \leqslant h \leqslant n-i$, so its corresponding gog triangle $H$ has

$$
H(n-i, k)=k+\sum_{m=0}^{k-1} s_{m} \quad \text { where } \quad 1 \leqslant k \leqslant n-i .
$$


The formulas in (8) and (9) are equivalent (taking $k=p+1$ ). Therefore, row $n-i$ of gog triangle $H$ contains the locations of the ones in the partial sum $\sum_{k=i+1}^{n} A_{k}$. In other words, gog triangle $H$ is constructed by considering the rows of alternating sign matrix $A$ in reverse order.

Proof of Theorem 6. Let $\gamma: \mathcal{G}_{n} \rightarrow \mathcal{G}_{n}^{\circ}$ be the bijection $\gamma(G)=G-G_{\text {min }}$. Let $\pi: \mathcal{G}_{n}^{\circ} \rightarrow$ $\triangle \mathcal{G}_{n}^{\circ}$ be the bijection $\pi\left(G^{\circ}\right)=\triangle G^{\circ}$. By Theorem 21, the desired involution $f: \mathcal{G}_{n} \rightarrow \mathcal{G}_{n}$ is $f=\gamma^{-1} \circ \pi^{-1} \circ \varphi \circ \pi \circ \gamma$.

\section{Conclusion and Future Work}

Poset refinements of the de Finetti Lattice $F_{n, 2}$ have interesting combinatorial connections. We have shown that $\mathcal{F}_{n, 2}$ is enumerated by the strict-sense ballot numbers and that $\mathcal{F}_{n, 2}^{1}$ is enumerated by the ASM/TSSCPP sequence. We have also shown that there is a very natural involution on gog triangles that corresponds to reversing the rows of the associated alternating sign matrices. We conclude this work with some open research questions relating to both poset refinement and ASM/TSSCPP.

One natural continuation of this work is to consider the de Finetti refinements of the order ideal $B_{n, m}$ for $3 \leqslant m \leqslant n$, with $m=3$ as the obvious starting point. Analogous to Section 1.2, let $F_{n, m}$ to be the unique minimal de Finetti refinement of $B_{n, m}$. For $3 \leqslant m \leqslant n$, let $\mathcal{F}_{n, m}$ denote the collection of linear extensions of $F_{n, m}$ that adhere to de Finetti's condition (F2). For $1 \leqslant k \leqslant m$, let $\mathcal{F}_{n, m}^{k}$ denote the collection of minimal de Finetti refinements of $F_{n, m}$ such that every set of size at most $k$ is comparable with all other sets. Are any of these families enumerated by known combinatorial sequences? If so, can we find a natural bijection to the appropriate combinatorial family?

An understanding of these poset families could provide valuable insight into the family $\mathcal{F}_{n}$ of de Finetti total orders. Any new perspective could have ramifications for comparative probability orders and completely separable preferences. One could further investigate the subfamily of de Finetti refinements $\mathcal{F}_{n, m}^{k}$ by defining a graph where we connect posets via an appropriately atomic operation, such as transpositions [26] for $\mathcal{L}\left(B_{n}\right)$ or flips [23] for members of $\mathcal{F}_{n}$. Also, can the dimension [35] of a de Finetti refinement of $F_{n, m}$ be achieved by restricting ourselves to de Finetti refinements?

This paper brings two novel families into the fold of ASM and TSSCPP combinatorial structures: the poset refinements $\mathcal{F}_{n, 2}^{1}$ and the kagog triangles $\mathcal{K}_{n}$. Some recent efforts have focused on statistic-preserving bijections between subfamilies of ASM and TSSCPP structures $[5,33]$. Perhaps the properties $\mathcal{F}_{n, 2}^{1}$ and $\mathcal{K}_{n}$ might reveal connections to help traverse the gap between ASM and TSSCPP. In particular, our two-color cube pyramid representation for triangular arrays revealed a natural bijection between magog triangles and kagog triangles, as well as a nice involution on gog triangles. We are optimistic that this point of view could aid in the investigation of the other known triangular families. 


\section{Acknowledgements}

We thank David Bressoud and Jessica Striker for sharing their insights about ASMs, TSSCPPs, triangular arrays and posets. We thank the anonymous referee for their suggestions for improving the exposition.

\section{References}

[1] G. Andrews. Plane partitions V: the TSSCPP conjecture. J. Combin. Theory, Ser. A, 66:28-39, 1994.

[2] A. Ayyer, R. Cori, and D. Gouyou-Beauchamps. Monotone triangles and 312 pattern avoidance. Electron. J. Comb., 18(2), 2011, \#P26.

[3] D. E. Barton and C. L. Mallows. Some aspects of the random sequence. Ann. Math. Statist., 36(1):236-269, 1965.

[4] A. Beveridge and I. Calaway. The voter basis and the admissibility of tree characters. arXiv: 1809.08332.

[5] P. Biane. Gog and magog triangles. In E. Celledoni, G. Di Nunno, K. EbrahimiFard, and H. Munthe-Kaas, editors, Computation and Combinatorics in Dynamics, Stochastics and Control, Abel Symposia, vol. 13, pages 99-127. Springer, 2018.

[6] W. J. Bradley, J. K. Hodge, and D. M. Kilgour. Separable discrete preferences. Math. Social Sci., 49(1):335-353, 2005.

[7] D. Bressoud and J. Propp. How the alternating sign matrix conjecture was solved. Notices of the AMS, 46(6):637-646, 1999.

[8] D. M. Bressoud. Proofs and Confirmations: The Story of the Alternating Sign Matrix Conjecture. Cambridge University Press, 1999.

[9] G. R. Brightwell and P. Tetali. The number of linear extensions of the boolean lattice. Order, 20:333-345, 2004.

[10] A. E. Brouwer and J. D. Christensen. Counterexamples to conjectures about subset takeaway and counting linear extensions of a boolean lattice. Order, 35:275-281, 2018.

[11] R. Christian, M. Conder, and A. Slinko. Flippable pairs and subset comparisons in comparative probability orderings. Order, 24:193-213, 2007.

[12] B. de Finetti. Sul significato soggettivo della probabilità. Fundamenta Mathematicae, 17:298-329, 1931.

[13] S. Felsner and M. Massow. Linear extension diameter of downset lattices of twodimensional posets. SIAM J. Discrete Math., 25:112-129, 2011.

[14] T. Fine and J. Gill. The enumeration of comparative probability relations. Ann. Prob., 4:667-673, 1976.

[15] J. Fink and P. Gregor. Linear extension diameter of level induced subposets of the boolean lattice. Eur. J. Comb., 35:221-231, 2014.

[16] P. C. Fishburn. Finite linear qualitative probability. J. Math. Psychology, 40:64-77, 1996. 
[17] P. C. Fishburn, A. Pekeč, and J. A. Reeds. Subset comparisons for additive linear orders. Math. Oper. Res., 27:227-243, 2002.

[18] J. K. Hodge. The mathematics of referendums and separable preferences. Math. Mag., 84(4):268-277, 2011.

[19] J. K. Hodge, M. Krines, and J. Lahr. Preseparable extensions of multidimmensional preferences. Order, 26(2):125-147, 2009.

[20] J. K. Hodge and M. TerHaar. Classifying interdependence in multidimensional binary preferences. Math. Social Sci., 55(2):190-204, 2008.

[21] C. H. Kraft, J. W. Pratt, and A. Seidenberg. Intuitive probability on finite sets. Ann. Math. Statist., 30:408-419, 1959.

[22] G. Kuperberg. Another proof of the alternating sign matrix conjecture. Int. Math. Research Notes, 1996:139-150, 1996.

[23] D. Maclagan. Boolean term orders and the root system $b_{n}$. Order, 15:279-295, 1999.

[24] W.H. Mills, D. P. Robbins, and H. Rumsey. Alternating-sign matrices and descending plane partitions. J. Combin. Theory, Ser. A, 34:340-359, 1983.

[25] J. Propp. The many faces of alternating-sign matrices. Discrete Math. and Th. Comp. Sci. Proc., AA (DM-CCG):43-58, 2001.

[26] G. Pruesse and F. Ruskey. Generating the linear extensions of certain posets by transpositions. SIAM J. Discrete Math, 4:413-422, 1991.

[27] J. C. Sha and D. J. Kleitman. The number of linear extensions of subset ordering. Discrete Math., 63:271-278, 1987.

[28] A. Slinko. Additive representability of finite measurement structures. In S. J. Brams, W. V. Gehrlein, and F. S. Roberts, editors, The Mathematics of Preference, Choice and Order, pages 113-133. Springer, 2009.

[29] N. J. A. Sloane. The On-Line Encyclopedia of Integer Sequences. https : //oeis . org. A003121, A046873, A005130 and A005806.

[30] R. P. Stanley. Enumerative Combinatorics, Volume I. Cambridge University Press, 1997.

[31] R. P. Stanley. Catalan Numbers. Cambridge University Press, 2015.

[32] J. Striker. A unifying poset perspective on alternating sign matrices, plane partitions, catalan objects. Adv. Appl. Math, 46(1-4):583-609, 2011.

[33] J. Striker. Permutation totally symmetric self-complementary plane partitions. Ann. Combin., 22(3):641-671, 2018.

[34] P. Terwilliger. A poset $\Theta$ whose maximal chains are in bijection with the $n \times n$ alternating sign matrices. Linear Alg. Appl., 554:79-85, 2018.

[35] W. T. Trotter. Combinatorics and Partially Ordered Sets: Dimension Theory. Johns Hopkins Series in the Mathematical Sciences. The Johns Hopkins University Press, 1992.

[36] D. Zeilberger. Proof of the alternating sign matrix conjecture. Electron. J. Comb., 3, 1996, \#R13 